\title{
Transfer Parcial para Extensões de Grupos Dualidades
}

\author{
Valdeni Soliani Franco.
}

orientador

Prof. Dr. Janey Antonio Daccach

Tese de doutorado apresentada ao Instituto de Ciências Matemáticas e de Computação, da Universidade de São Paulo, como parte dos requisitos para obtenção do título de "Doutor em Ciências - Área Matemática - Geometria e Topologia".

\section{USP - Sāo Carlos}




\section{Agradecimentos}

Ao Professor Janey Antonio Daccach pela dedicação, estímulo e amizade.

À Carla pelo apoio implícito por alguns anos e explícito neste último ano.

Ao Rafael pela compreensão que teve com as longas ausências às quais fui obrigado a submetê-lo.

Aos meus pais pela força e fé.

Aos professores do DMA-UEM pelas condições e incentivo que me deram durante estes anos.

Aos professores do ICMC-USP, pela formação e apoio.

Aos funcionários do DMA-UEM e ICMC-USP que nos deram o suporte necessário para conclusão deste trabalho.

Aos companheiros de caronas e repúblicas pela boa convivência nestes anos.

Enfim a todos os amigos que acreditaram, torceram, me incentivaram, e que de alguma forma contribuiram para a realização deste trabalho.

A CAPES e ao CNPq pelo auxílio financeiro.

A Deus que mais uma vez me mostrou sua cara. 
ao Rafael 


\section{Abstract}

Since the concept of Poincaré Duality Groups was created, many efforts has been done in order to study the geometrical properties of a closed manifold, in this new category. This is natural because a Poincaré Duality Group has its homology and cohomology connected by an isomorfism like the Poincaré Duality isomorphism for compact manifolds.

Products like the cup and cap product, Steenrod square operations, universal coeficient theorems can also be defined for Poincaré Duality Groups. In this way StiefelWhitney classes, Stiefel Whitney numbers and the notion of cobordism of Poincaré Duality Groups can be naturally established. Also the notion of index of Poincaré Duality Group can be defined, and since its properties are essentially algebraic, the results in this direction would not be new. For completness we prove here the multiplicative property of the index for "fibrations" (under cetain hipothesis) and we give counter-examples like the ones given by Atiyah and Kodaira.

The core of this work is the Partial Transfer Theorem of Gotllieb. His proof uses deep geometrical properties of manifolds, still without similar for Poincaré Duality Groups. Thanks for LHS spectral sequence we present here a proof of the Partial Transfer Theorem for a short exact sequence:

$$
N \stackrel{i}{\rightarrow} G \stackrel{\pi}{\rightarrow} Q
$$

of Poincaré Draality Groups and some applications is given. 


\section{Resumo}

Desde que o conceito de Grupos Dualidades de Poincaré foi criado, muitos esforços tem sido feito com a finalidade de estudar as propriedades geométricas de uma variedade fechada nesta nova categoria. Isto é natural, pois um Grupo Dualidade de Poincaré tem sua homologia e cohomologia conectada por um isomorfismo semelhante ao isomorfismo Dualidade Poincaré para variedades compactas.

Produtos semelhantes ao cup e cap, operações quadrados de Steenrod, Teorema dos Coeficientes Universais também podem ser definidos para Grupos Dualidade de Poincaré. Da mesma maneira as classes de Stiefel-Whitney, números de StiefelWhitney e a noção de cobordismo de Grupos Dualidade de Poincaré pode ser definido, e desde que estas propriedades são essencialmente algébricas, os resultados nesta direção não são novos. Apenas como complemento nós demonstraremos aqui propriedades multiplicativos do índice de "fibrações" (sob certas hipóteses) e damos contra-exemplos semelhantes aos dados por Atiyah e Kodaira.

O centro deste trabalho é o Teorema de Transfer Parcial de Gotllieb. Sua demonstração utiliza propriedades geométricas refinadas de variedades, que não tem similares para Grupos Dualidades de Poincaré. Graças a sequência espectral de LHS, nós apresentamos aqui uma demonstração do Teorema de Transfer Parcial para uma sequência exata curta:

$$
N \stackrel{i}{\rightarrow} G \stackrel{\pi}{\rightarrow} Q
$$

de Grupos Dualidades de Poincaré e damos algumas aplicações. 


\section{ÍNDICE}

CAPÍTULO 0: ENUNCIADO E DISCUSSÃO DO PROBLEMA APRE-

SENTADO NESTE TRABALHO.

CAPÍTULO I: (CO)HOMOLOGIA DE GRUPOS E GRUPOS DUALI-

DADES.

I.1 - Homologia e cohomologia de Grupos.

I.1.1 - Anel de Grupo

I.1.2 - Teoremas de álgebra homológica 06

I.1.3 - Observações sobre Módulos. .07

I.1.4 - Resoluções projetivas. .08

I.1.5 - As definições de homologia e cohomologia de Grupos. .11

I. 1.6 - Extensão e Coextensão de escalares. .14

I. 2 - Homologia e Cohomologia relativa para pares de Grupos. 16

I.2.1 - Definições. 16

I. 2.2 - Resultado básico .17

I.3 - O produto Cap absoluto e relativo .18

I.3.1 - Definições e notações. .19

I.3.2 - O produto cap rela tivo. .22 
I.4 - Grupos dualidades e pares dualidades.

I.4.1 - Os grupos dualidades e grupos dualidades de Poincaré 23

I.4.2 - Condições de finitude .25

I.4.3 - Pares dualidades e pares dualidades de Poincaré 27

I.5 - O produto Cup. .28

I.5.1 - O produto cross. .29

I.5.2 - O produto cup 29

I.6 - O grau de um homomorfismo 31

I.6.1 - Definições. 31

I.6. 2 - Resultados e exemplos. .33

I.7 - O índice de uma extensão de grupos. .37

CAPÍTULO II : UMA FÓRMULA PARA EXTENSÃO DE GRUPOS 40

II.1 - As sequências espectrais de LHS e a compatibilidade com o produto Cap. .42

II. 2 - Um teorema básico. .44

II.3 - A demonstração do teorema principal. .50

CAPÍTULO III : O GRAU DE UMA APLICAÇÃO E O NÚMERO DE UMA EXTENSÃO. 52 


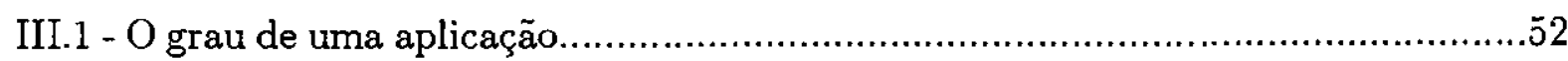

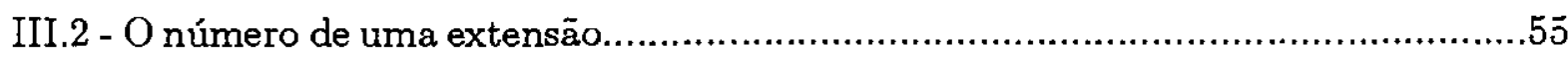

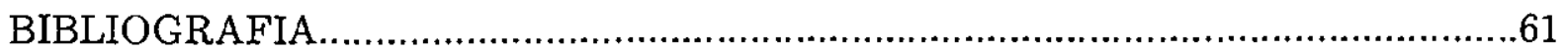




\section{CAPÍTULO 0}

\section{ENUNCIADO E DISCUSSÃO DO PROBLEMA APRESENTADO NESTE TRABALHO}

Um dos conceitos importantes em teoria de homotopia é o conceito de fibração.

A definição dos grupos de homotopia de um espaço X é relativamente simples se comparado com a definição de grupos de homologia do espaço $\mathrm{X}$.

Uma vez estabelecido o conceito de homologia o cálculo dos grupos de homogia para determinadas categorias, como por exemplo CW-complexos, nada mais é que uma rotina em geral.

No entanto, o cálculo dos grupos de homotopia, apesar de sua simples definição, é muitíssimo complicado pois não temos em homotopia um conceito equivalente a sequência de Mayer-Vietoris.

Por exemplo, na década de 30 , ainda não se sabia calcular $\pi_{3}\left(S^{2}\right)$, sem desmerecer o grande matemático deste século Pontryagin, ele erroneamente enunciou que $\pi_{3}\left(S^{2}\right)$ era o grupo trivial.

Foi Hopf logo em seguida que estudando a fibração: 


$$
\begin{array}{r}
S^{1} \hookrightarrow S^{3} \\
{ }^{\pi} \downarrow \\
S^{2}
\end{array}
$$

que recebeu seu nome, conseguiu concluir com sucesso que $\pi_{3}\left(S^{2}\right)=\mathbb{Z}$ cujo gerador é a classe de homotopia da função $\pi$.

Desde então o conceito de fibração tem desempenhado papel fundamental na teoria de homotopia.

De forma alguma é nosso interesse neste capítulo fazer uma exposição exaustiva das propriedades das fibrações nas diferentes categorias, ou seja: base, fibra e espaço total como sendo CW-complexos, ou base, fibra, espaço total, variedades, etc.

Duas propriedades interessantes relativas a fibrações (na categoria de variedades que suporemos diferenciáveis) que deu origem a este trabalho são as seguintes:

TEOREMA A (Gottlieb): Para uma variedade fibrada:

$$
\begin{array}{r}
F \stackrel{i}{\leftrightarrow} E \\
{ }^{p} \downarrow \\
B
\end{array}
$$

a seguinte fórmula é verificada:

$$
i_{*}([F])=\left(p^{*}[\bar{B}]\right) \frown[E] \text {, onde }[E] \in \mathrm{H}_{n+m}(E),[F] \in \mathrm{H}_{n}(F) \text { são classes }
$$

fundamentais e $[\bar{B}]$ a classe fundamental dual em $\mathrm{H}^{m}(B)$. 
TEOREMA B (Chern-Hirzebruch-Serre): Consideremos uma variedade fibrada:

$$
\begin{array}{r}
F \stackrel{i}{\leftrightarrow} E \\
{ }^{p} \downarrow \\
B
\end{array}
$$

satisfazendo as seguintes condições:

(1) $E, B$ e $F$ são variedades compactas conexas e orientáveis,

(2) $\mathrm{O}$ grupo fundademental $\pi_{1}(B)$ atua trivialmente na cohomologia $\mathrm{H}^{*}(F)$.

Então $E, B$ e $F$ são coerentemente orientadas, e o índice de $E$ é o produto dos índices de $\mathrm{F}$ e $\mathrm{B}$, isto é:

$$
\sigma(E)=\sigma(F) \sigma(B)
$$

Um conceito em teoria de grupos que nos parece similar ao conceito de fibração, é o da sequência exata curta de grupos:

$$
N \mapsto G \rightarrow Q
$$

Uma vez que existe uma determinada classe de grupos, chamada grupos dualidades, cujas homologia e cohomologia satisfazem uma propriedade semelhante ao das variedades compactas; ou seja dualidade de Poincaré, procuramos neste trabalho demonstrar nesta categoria os dois teoremas acima enunciados.

Na demonstração do Teorema A (diga-se de passagem resultado talvez já conhecido por Hopf, porém sem referencias na literatura) Gottlieb usou técnicas 
refinadas de topologia diferencial. No entanto até o momento não possuímos para grupos, técnicas semelhantes, como por exemplo transversalidade, fibrado normal e outras.. Isto que tornou atrativo o estudo do Teorema A na categoria de Grupos Dualidade de Poincaré.

Com relação ao Teorema B sua demonstração baseia-se em propriedades dos diferentes produtos e de sequências espectrais. No entanto temos na categoria de grupos todos estes conceitos gozando das mesmas propriedades algébricas. Assim sendo, a demonstração no caso de Grupos Dualidade nada mais é que uma transposição do trabalho de Chern-Hirzebruch-Serre para o caso de uma sequência curta exata de Grupos Dualidade:

$$
N \mapsto G \rightarrow Q
$$

Ainda com relação ao teorema B que pelo motivo acima exposto, somente enunciaremos no final do capítulo I, vamos da mesma maneira que Atiyah[2] ou Kodaira[21], exibir um contra-exemplo onde o teorema falha se o grupo fundamental da base não atua trivialmente na cohomologia da fibra, entendendo obviamente no caso de uma sequência exata de grupos como acima, $Q$ atuando trivialmente na cohomologia de $N$. Isto será apresentado unicamente para reforçar uma conjectura em aberto, que diz que todo Grupo Dualidade é o grupo fundamental de uma variedade, fato este que já foi demonstrado para Grupos Dualidade de dimensão 2, ou seja:

Todo Grupo Dualidade de dimensão 2 é grupo fundamental de uma superfície de genus diferente de zero [14]. 


\section{CAPÍTULO I}

\section{(CO)HOMOLOGIA DE GRUPOS E GRUPOS DUALIDADES}

Aqui pretendemos estabelecer notações, introduzir conceitos, enunciar e se necessário demonstrar resultados que utilizaremos no decorrer deste trabalho. Também definimos e damos alguns resultados importantes de (co)homologia relativa de pares de grupos que estão servindo de base para pesquisas recentes que estamos desenvolvendo e que não farão parte deste trabalho.

Aqui G denotará um grupo qualquer e $\mathrm{K}$ um anel com unidade.

\section{I.1 - HOMOLOGIA E COHOMOLOGIA DE GRUPOS}

Nesta seç̧ão após algumas definições e propriedades básicas, definiremos o conceito fundamental para o desenvolvimento deste trabalho e também alguns resultados que serão utilizados nas seç̧ões e capítulos posteriores.

\section{I.1.1 - Anel de Grupo}

O anel de grupo KG é o anel cujos elementos são todas as somas formais $\lambda=\sum_{g \in G} \lambda_{g} g \quad, \lambda_{g} \in \mathrm{K}$, tal que: 
$\operatorname{suporte}(\lambda)=\left\{g ; \lambda_{g} \neq 0\right\}$ é finito e com as seguintes operações:

(1) $\sum_{g \in G} \lambda_{g} g+\sum_{g \in G} \mu_{g} g=\sum_{g \in G}\left(\lambda_{g}+\mu_{g}\right) g$

(2) $\left(\sum_{g \in G} \lambda_{g} g\right)\left(\sum_{g \in G} \mu_{g} g\right)=\sum_{g \in G} \nu_{g} g, \quad$ onde $\nu_{g}=\sum_{g \in G} \lambda_{h} \mu_{h^{-1} g}=\sum_{x y=g} \lambda_{x} \mu_{y}$

Eliminando as componentes nulas da soma fomal, $\lambda$ pode ser escrito como:

$$
\lambda=\sum_{i=1}^{n} \lambda_{i} g_{i}
$$

Assim $k \rightarrow k e_{G}$ é um mergulho de $\mathrm{K}$ em KG. Após a identificação de $\mathrm{K}$ com $\mathrm{Ke}_{G}$ podemos assumir que $\mathrm{K}$ está contido em $\mathrm{KG}$.

O elemento $1 . e_{G}$ atua como identidade para $\mathrm{KG}$ e nós o denotaremos por 1, como usual.

A aplicação $\varepsilon: \mathrm{KG} \rightarrow \mathrm{K}$, tal que $\varepsilon\left(\sum_{g \in G} \lambda_{g} g\right)=\sum_{g \in G} \lambda_{g}$ é um homomorfismo chamado aplicaçāo aumentação de KG.

Seu kernel $\Delta_{K}(\mathrm{G})=\left\{\lambda=\sum_{g \in G} \lambda_{g} g \in \mathrm{KG} ; \sum_{g \in G} \lambda_{g}=0\right\}$ é chamado o ideal aumentaçāo de KG.

\section{I.1.2 - Teoremas de álgebra homológica}

Teorema (Fórmula de Künneth): Seja K um domínio ideal principal e seja $C$ e $C^{\prime}$ complexos de cadeiais tal que $C$ é livre em cada dimensão. Existem sequências exatas naturais:

$0 \rightarrow \bigoplus_{p \in \mathbb{Z}} \mathrm{H}_{p}(C) \otimes_{K} \mathrm{H}_{n-p}\left(C^{\prime}\right) \rightarrow \mathrm{H}_{n}\left(C \otimes_{K} C^{\prime}\right) \rightarrow \bigoplus_{p \in \mathbf{Z}} \operatorname{Tor}_{1}^{K}\left(\mathrm{H}_{p}(C), \mathrm{H}_{n-p-1}\left(C^{\prime}\right)\right) \rightarrow 0$ e

$0 \rightarrow \prod_{p \in \mathbb{Z}} \operatorname{Ext}_{K}^{1}\left(\mathrm{H}_{p}(C), \mathrm{H}_{n-p+1}\left(C^{\prime}\right)\right) \rightarrow \mathrm{H}_{n}\left(\operatorname{Hom}_{K}\left(C, C^{\prime}\right)\right) \rightarrow \prod_{p \in \mathbb{Z}} \operatorname{Hom}_{K}\left(\mathrm{H}_{p}(C), \mathrm{H}_{n-p}\left(C^{\prime}\right)\right) \rightarrow 0$ e estas sequências splitam. 
Um caso especial da fórmula acima é dado pelo

Teorema dos Coeficientes Universais(TCU): Com as hipóteses do teorema acima e supondo que $C^{\prime}$ é tal que $C_{0}^{\prime}=M$ e $C_{n}^{\prime}=0$ para $n \neq 0$, teremos que as sequências exatas neste caso tomam a seguinte forma:

$$
\begin{aligned}
& 0 \rightarrow \mathrm{H}_{p}(C) \otimes_{K} M \rightarrow \mathrm{H}_{n}\left(C \otimes_{K} M\right) \rightarrow \bigoplus_{p \in \mathbb{Z}} \operatorname{Tor}_{1}^{K}\left(\mathrm{H}_{n-1}(C), M\right) \rightarrow 0 \text { e } \\
& 0 \rightarrow \operatorname{Ext}_{K}^{1}\left(\mathrm{H}_{n-1}(C), M\right) \rightarrow \mathrm{H}_{n}\left(\operatorname{Hom}_{K}(C, M)\right) \rightarrow \operatorname{Hom}_{K}\left(\mathrm{H}_{n}(C), M\right) \rightarrow 0
\end{aligned}
$$

\section{I.1.3 - Observações sobre Módulos}

Neste trabalho, a menos que se especifique o contrário, trabalharemos na categoria de KG-módulos à esquerda. Mas é bom observar que todo KG-módulo à esquerda $M$ pode ser considerado um KG-módulo à direita bastando definir:

$$
m(k g)=\left(k g^{-1}\right) m \quad \forall k \in \mathrm{K}, g \in \mathrm{G} \text { e } m \in M .
$$

De modo análogo todo KG-módulo à direita pode ser considerado KGmódulo à esquerda.

Se $M$ é um KG-módulo, então:

(i) $M$ é um K-módulo (basta definir $k m=(k e) m$ ) onde $e$ é o elemento neutro de G.

(ii) Existe uma ação de $\mathrm{G}$ em $M$, dada por :

$$
g * m=(1 g) m
$$

Podemos estabelecer a recíproca, ou seja, se $M$ é um K-módulo e existe uma ação de $\mathrm{G}$ em $M$, então podemos dar a $M$ uma estrutura de KG-módulo, bastando definir: 


$$
(k g) * m=k(g m)
$$

e estender por linearidade.

$\mathrm{O}$ anel $\mathrm{K}$ pode ser sempre considerado como um KG-módulo com a G-ação trivial, isto é, $g * k=k, \forall k \in \mathrm{K} \mathrm{e} \forall g \in \mathrm{G}$.

$$
\text { Assim }\left(\sum_{g \in G} k_{g} g\right) k=\sum_{g \in G} k_{g}(g * k)=\sum_{g \in G} k_{g} k .
$$

Sejam $M$ e $N$ KG-módulos. Podemos dar a $M \otimes_{K} N$ e a $\operatorname{Hom}_{K}(M, N)$ uma estrutura de KG-módulo induzida pela G-ação diagonal, como segue:

$$
\begin{aligned}
& g *(m \otimes n)=g m \otimes g n, \forall g \in \mathrm{G}, \forall m \in M \text { e } \forall n \in N . \\
& (g * f)(m)=g f\left(g^{-1} m\right), \forall g \in \mathrm{G}, \forall m \in M \text { e } \forall f \in \operatorname{Hom}_{K}(M, N) .
\end{aligned}
$$

\section{I.1.4 - Resoluções projetivas}

Uma resoluçāo projetiva de $\mathbf{K}$ como $\mathbf{K G}$-módulos é uma seqüência exata de KG-módulos:

$$
\cdots \longrightarrow P_{n} \stackrel{\partial_{n}}{\longrightarrow} P_{n-1} \longrightarrow \cdots \longrightarrow P_{1} \stackrel{\partial_{1}}{\longrightarrow} P_{0} \stackrel{\epsilon}{\longrightarrow} K \longrightarrow 0
$$

onde cada $P_{i}$ é KG-projetivo.

A existência de uma tal projeção é óbvia, basta tomar $P_{0}=\mathrm{KG}, \varepsilon$ a aplicação aumentação e definir $P_{n+1}$ como um módulo livre qualquer que aplica sobre $\mathrm{K}_{n}=\operatorname{ker}\left(\partial_{n}: P_{n} \longrightarrow P_{n-1}\right)$ e para $\partial_{n+1}$ a aplicação composta :

$$
P_{n+1} \rightarrow \mathrm{K}_{n} \hookrightarrow P_{n} .
$$

Observemos que na verdade esta construção nos dá uma resolução livre de K.

As vezes denotamos uma resolução projetiva de $\mathrm{K}$, simplesmente por : 


$$
\mathrm{P} \rightarrow \mathrm{K} .
$$

*Observações:

No estudo da (co)homologia de um grupo G, utilizamos resoluções projetivas de $\mathbb{Z}$ de $\mathbb{Z} G$-módulos, conforme veremos no próximò parágrafo.

Em $[10,1.7 .4]$ mostra-se o seguinte teorema:

Teorema 1: Dadas duas resoluções projetivas $\mathbf{P}$ e $\mathbf{P}^{\prime}$ de um módulo $M$, existe uma aplicação $f: \mathbf{P} \longrightarrow \mathbf{P}^{\prime}$, única a menos de homotopia e $f$ é uma eqüivalência homotópica.

Devido a observação acima e a este último teorema será interessante exibir uma resolução projetiva de $\mathbb{Z}$ bastante importante no estudo da (co)homologia de grupos, denominada resoluçāo Bar.

Com esse objetivo daremos inicialmente uma resoluçāo padrão.

É uma resolução de $\mathbb{Z}$ como $\mathbb{Z} G$-módulos

Consideramos $P_{n}$ como o grupo abeliano livre gerado por $\mathrm{G}^{n+1}=\underbrace{\mathrm{G} \times \ldots \times \mathrm{G}}_{n+1}$

Se $x \in P_{n} \Rightarrow x=\sum_{i} n_{i}\left(x_{0}^{i}, x_{1}^{i}, \ldots, x_{n}^{i}\right)$ onde $n, i \in \mathbb{Z}$.

Vamos definir uma ação à esquerda de $\mathrm{G}$ em $P_{n}$ :

$\mathrm{G} \times P_{n} \longrightarrow P_{n}$, tal que $\left(x,\left(x_{0}, \ldots, x_{n}\right)\right) \mapsto\left(x x_{0}, \ldots, x x_{n}\right)$.

Definimos ainda $\partial_{n+1}: P_{n+1} \longrightarrow P_{n}$, colocando:

$\partial_{n+1}\left(x_{0}, \ldots, x_{n+1}\right)=\sum_{i}(-1)^{i}\left(x_{0}, \ldots, \dot{x}_{i}, \ldots, x_{n+1}\right)$

$\cdots \longrightarrow P_{n} \stackrel{\partial_{n}}{\longrightarrow} P_{n-1} \longrightarrow \cdots \longrightarrow P_{1} \stackrel{\partial_{1}}{\longrightarrow} P_{0} \stackrel{\varepsilon}{\longrightarrow} \mathbb{Z} \longrightarrow 0$ 
Mostra-se que a resolução padrão é uma resolução livre de $\mathbb{Z}$ de $\mathbb{Z} G$-módulos. Vamos agora definir a resolução Bar

As cadeias $\mathrm{B}_{n}$ desta resolução serão os $\mathbb{Z} \mathrm{G}$-módulos gerado por $\mathrm{G}^{n}$.

Um gerador de $\mathrm{B}_{n}$ é da forma: $\left[x_{1}|\ldots| x_{n}\right]$, onde $x_{i} \in \mathrm{G}$.

Com o objetivo de definir os homomorfismos $\bar{\partial}_{n}: \mathrm{B}_{n} \longrightarrow \mathrm{B}_{n-1}$, vamos encontrar uma correspondência biunívoca entre $P_{n}$ e $\mathrm{B}_{n}$.

$\cdots \longrightarrow P_{n+1} \stackrel{\partial_{n+1}}{\longrightarrow} P_{n} \stackrel{\partial_{n}}{\longrightarrow} P_{n-1} \longrightarrow \cdots \longrightarrow P_{1} \stackrel{\partial_{1}}{\longrightarrow} P_{0} \stackrel{\varepsilon}{\longrightarrow} \mathbb{Z} \longrightarrow 0$

$$
\tau_{n} \mid \uparrow \sigma_{n}
$$

$\cdots \longrightarrow \mathrm{B}_{n+1} \stackrel{\bar{\partial}_{n+1}}{\longrightarrow} \mathrm{B}_{n} \stackrel{\bar{\partial}_{n}}{\longrightarrow} \mathrm{B}_{n-1} \longrightarrow \cdots \longrightarrow \mathrm{B}_{1} \stackrel{\bar{\partial}_{1}}{\longrightarrow} \mathrm{B}_{0} \stackrel{\varepsilon}{\longrightarrow} \mathbb{Z} \longrightarrow 0$

Definimos então:

$$
\begin{aligned}
& \tau_{n}\left(x_{0}, \ldots, x_{n}\right)=x_{0}\left[x_{0}^{-1} x_{1}\left|x_{1}^{-1} x_{2}\right| \ldots \mid x_{n-1}^{-1} x_{n}\right] \\
& \sigma_{n}\left[x_{1}|\ldots| x_{n}\right]=\left(1, x_{1}, x_{1} x_{2}, x_{1} x_{2} x_{3}, \ldots, x_{1} x_{2} \ldots x_{n}\right)
\end{aligned}
$$

Observamos que $\sigma_{n} \tau_{n}\left(x_{0}, \ldots, x_{n}\right)=i d\left(x_{0}, \ldots, x_{n}\right)$ e que $\tau_{n} \sigma_{n}\left[x_{1}|\ldots| x_{n}\right]=$ $i d\left(\left[x_{1}|\ldots| x_{n}\right]\right)$

Temos:

$$
\begin{aligned}
& \cdots \longrightarrow P_{n+1} \stackrel{\partial_{n+1}}{\longrightarrow} P_{n} \stackrel{\partial_{n}}{\longrightarrow} P_{n-1} \longrightarrow \cdots \\
& \sigma_{n} \uparrow \downarrow \downarrow^{\tau_{n-1}} \\
& \cdots \longrightarrow \mathrm{B}_{n+1} \stackrel{\bar{\partial}_{n+1}}{\longrightarrow} \mathrm{B}_{n} \stackrel{\bar{\partial}_{n}}{\longrightarrow} \mathrm{B}_{n-1} \longrightarrow \cdots
\end{aligned}
$$

Definimos então: $\bar{\partial}_{n}=\tau_{n-1} \partial_{n} \sigma_{n}$. Desta maneira podemos dar uma expressão para $\bar{\partial}_{n}$ como segue: 
$\bar{\partial}_{n}\left[x_{1}|\ldots| x_{n}\right]=x_{1}\left[x_{2}\left|x_{3}\right| \ldots \mid x_{n}\right]+\sum_{i=1}^{n-1}(-1)^{i}\left[x_{1}\left|x_{2}\right| \ldots x_{i} x_{i+1}|\ldots| x_{n}\right]+$

$+(-1)^{n}\left[x_{1}|\ldots| x_{n-1}\right]$.

* Notação: $\mathrm{B}(\mathrm{G})$ é a resolução bar de $\mathrm{G}$.

I.1.5 - As definições de homologia e cohomologia de grupos

Aqui consideraremos $\mathbb{Z}$ como um $\mathbb{Z G}$-módulo trivial.

Tomemos uma $\mathbb{Z}$ G-resolução projetiva de $\mathbb{Z}$ :

$\cdots \longrightarrow P_{n} \stackrel{\partial_{n}}{\longrightarrow} P_{n-1} \stackrel{\partial_{n-1}}{\longrightarrow} \cdots \stackrel{\partial_{2}}{\longrightarrow} P_{1} \stackrel{\partial_{1}}{\longrightarrow} P_{0} \stackrel{\varepsilon}{\longrightarrow} \mathbb{Z} \longrightarrow 0$.

Construímos as sequências abaixo, onde $M$ é um $\mathbb{Z} G$-módulo dado:

$\ldots \longrightarrow P_{\mathrm{n}} \otimes_{\mathrm{ZG}} M^{\theta_{n} \otimes I d} P_{\mathrm{n}-1} \otimes_{\mathrm{ZG}} M \stackrel{\theta_{\mathrm{n}-1} \otimes I d}{\longrightarrow} \ldots \stackrel{\theta_{2} \otimes I d}{\longrightarrow} P_{1} \otimes_{\mathrm{ZG}} M \stackrel{\theta_{1} \otimes I d}{\longrightarrow} P_{0} \otimes_{\mathrm{ZG}} M \stackrel{\& \otimes I d}{\longrightarrow} Z \otimes_{\mathrm{ZG}} M \longrightarrow 0$

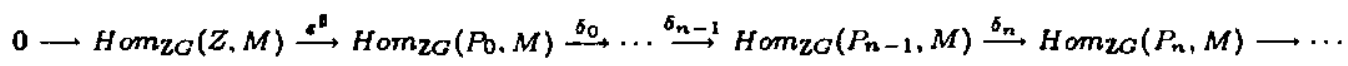

Aqui $\delta_{i}$ é definido como segue:

Se $f \in H o m_{\mathbb{Z} G}\left(P_{n}, M\right) \Rightarrow\left(\delta_{n}(f)\right)(x)=(-1)^{n+1} f\left(\partial_{n+1}(x)\right), \forall x \in P_{n+1}$.

Por definição, a homologia de $\mathbf{G}$ com coeficientes em $\mathbf{M}\left(\mathrm{H}_{*}(G ; M)\right)$, é a homologia do complexo:

$\ldots \longrightarrow P_{n} \otimes \mathrm{ZG} M \stackrel{\partial_{n} \otimes I d}{\longrightarrow} P_{n-1} \otimes \mathrm{ZG} M \stackrel{\partial_{n-1} \otimes I d}{\longrightarrow} \ldots \stackrel{\partial_{2} \otimes I d}{\longrightarrow} P_{1} \otimes_{\mathrm{ZG}} M \stackrel{\partial_{1} \otimes I d}{\longrightarrow} P_{0} \otimes \mathrm{ZG} M \longrightarrow 0$

e a cohomologia de $\mathbf{G}$ com coeficientes em $\mathbf{M}\left(\mathrm{H}^{*}(G ; M)\right)$, é a homologia do complexo:

$0 \longrightarrow \operatorname{Hom}_{\mathbf{Z} G}\left(P_{0}, M\right) \stackrel{\delta_{0}}{\longrightarrow} \operatorname{Hom}_{\mathbf{Z} G}\left(P_{1}, M\right) \stackrel{\delta_{1}}{\longrightarrow} \cdots \stackrel{\delta_{n-1}}{\longrightarrow} \operatorname{Hom}_{\mathbf{Z} G}\left(P_{n-1}, M\right) \stackrel{\delta_{n}}{\longrightarrow} \operatorname{Hom}_{\mathbf{Z} G}\left(P_{n}, M\right) \longrightarrow$ 
Podemos calcular facilmente $\mathrm{H}_{0}(\mathrm{G} ; M)=M_{G} \cong Z \otimes \mathbf{Z} G M$ e $\mathrm{H}^{0}(\mathrm{G} ; M)=$ $M^{G}=\{m \in M ; g m=m, \forall g \in \mathrm{G}\}$.

${ }^{*}$ Notações especiais:

1. Quando fazemos $M=\mathbb{Z}$, denotamos $\mathrm{H}_{*}(\mathrm{G}, \mathbb{Z})$ e $\mathrm{H}^{*}(\mathrm{G}, \mathbb{Z})$ simplesmente por $H_{*}(G)$ e $H^{*}(G)$, respectivamente.

2. Os funtores $\operatorname{Hom}_{\mathbf{Z}(-, .)}, \operatorname{Hom}_{\mathrm{Z} G}(-,-)_{,-} \otimes_{\mathrm{Z}-}$ e $-\otimes_{\mathrm{Z} G-}$ serão denotados, respectivamente, por $\operatorname{Hom}(-,),, \operatorname{Hom}_{G}(-,-), \otimes_{-} \otimes_{-} \otimes_{G-}$.

* Observação:

No teorema dos coeficientes universais I.1.2, se $\mathrm{K}$ é um corpo, os termos Tor e Ext se anulam e assim:

$$
\begin{aligned}
& \mathrm{H}_{*}(\mathrm{G} ; M) \cong \mathrm{H}_{*}(\mathrm{G} ; K) \otimes M \mathrm{e} \\
& \mathrm{H}^{*}(\mathrm{G} ; M) \cong \operatorname{Hom}\left(\mathrm{H}_{*}(\mathrm{G} ; \mathrm{K}), M\right) \\
& \mathrm{H}^{*}(\mathrm{G} ; M) \cong \mathrm{H}^{*}(\mathrm{G} ; \mathrm{K}) \otimes M
\end{aligned}
$$

este fato encontra-se demonstrado em $[16, \S 3.4]$.

Temos uma caracterização topológica dos grupos de homologia e cohomologia $H_{*}(G)$ e $H^{*}(G)$ dada em $[10, I I, \S 4$, prop. 4.1$]$

Se $\mathbf{Y}$ é um $K(G, 1)$-complexo, então $H_{*}(G) \cong H_{*}(\mathbf{Y})$ e $H^{*}(G) \cong H^{*}(\mathbf{Y})$.

Como aplicações deste resultado temos:

1. $\mathrm{H}_{n}(\mathbb{Z})=\left\{\begin{array}{c}\mathbb{Z}, \text { se } n=0,1 \\ 0, \quad \text { se } n>1\end{array} \quad K(Z, 1) \cong S^{1}\right.$ 
2. Seja $G_{n}$ o grupo fundamental de uma superfície orientável $M_{n}^{2}$ de genus $n$, $n>0 \Rightarrow \mathrm{H}_{*}\left(\mathrm{G}_{n}\right)=\mathrm{H}_{*}^{s}\left(\mathrm{M}_{n}^{2}, \mathbb{Z}\right)$

De Fato: Tomamos o recobrimento universal de $\mathrm{M}_{n}, \tilde{\mathrm{M}}_{n}$. Como a cardinalidade de $\pi_{1}\left(M_{n}^{2}\right)$ é infinita, cada ponto de $M_{n}$ possui uma vizinhança admissível que se levanta em infinitas folhas. Portanto $\widetilde{\mathrm{M}}_{n}$ não é compacto. Logo $\mathrm{H}_{2}\left(\widetilde{\mathrm{M}}_{n}\right)=0$ e como $\mathrm{H}_{i}\left(\tilde{\mathrm{M}}_{n}\right)=0$ para $i>2$, pois $\operatorname{dim} \tilde{\mathrm{M}}_{n}=2, \pi_{1}\left(\tilde{\mathrm{M}}_{n}\right)=0$ pois o recobrimento é universal.

O teorema de Hurewicz garante que $\pi_{2}\left(\tilde{\mathrm{M}}_{n}\right)=\mathrm{H}_{2}\left(\tilde{\mathrm{M}}_{n}\right)=0$ e $\pi_{1}\left(\tilde{\mathrm{M}}_{n}\right)=$ 0 . Portanto $\widetilde{\mathrm{M}}_{n}$ é contrátil e assim $\mathrm{M}_{n}$ é um $\mathrm{K}\left(\mathrm{G}_{n}, 1\right)$. Pelo resultado acima $\mathrm{H}_{*}\left(\mathrm{G}_{n}\right)=\mathrm{H}_{*}^{s}\left(\mathrm{M}_{n}^{2}, \mathbb{Z}\right)$.

No caso dos grupos $\mathrm{H}_{*}(\mathrm{G} ; M)$ e $\mathrm{H}^{*}(\mathrm{G} ; M)$ onde $M$ é um $\mathbb{Z} \mathrm{G}$-módulo qualquer, a interpretação topológica é análoga e vamos enunciá-la como teorema, pois a utilizaremos em capítulos posteriores.

Teorema $1[10, \operatorname{pag} 59]: \mathrm{H}_{*}(\mathrm{G} ; M) \cong \mathrm{H}_{*}(\mathrm{~K}(\mathrm{G}, 1) ; \mathfrak{M})$ e $\mathrm{H}^{*}(\mathrm{G} ; M) \cong \mathrm{H}^{*}(\mathrm{~K}(\mathrm{G}, 1) ; \mathfrak{M})$, onde $\mathfrak{M}$ é o sistema de coeficientes locais de $K(G, 1)$ associado ao G-módulo $M$.

Teorema 2: Seja $\mathrm{G}$ um grupo qualquer e $M$ um $\mathbb{Z} G$-módulo. Então para qualquer sequência exata curta $0 \rightarrow M^{\prime} \rightarrow M \rightarrow M^{\prime \prime} \rightarrow 0$ de $\mathbb{Z} G$-módulos e qualquer inteiro $n$, existem homomorfismos naturais:

$$
\partial: \mathrm{H}_{n}\left(\mathrm{G} ; M^{\prime \prime}\right) \longrightarrow \mathrm{H}_{n-1}\left(\mathrm{G} ; M^{\prime}\right) \text { e } \delta: \mathrm{H}^{n}\left(\mathrm{G} ; M^{\prime \prime}\right) \longrightarrow \mathrm{H}^{n+1}\left(\mathrm{G} ; M^{\prime}\right) \text { tais que }
$$
as sequências longas:

$\cdots \stackrel{p_{*}}{\longrightarrow} \mathrm{H}_{n}\left(\mathrm{G} ; M^{\prime \prime}\right) \stackrel{\partial}{\longrightarrow} \mathrm{H}_{n-1}\left(\mathrm{G} ; M^{\prime}\right) \stackrel{2_{*}}{\longrightarrow} \mathrm{H}_{n}(\mathrm{G} ; M) \stackrel{p_{*}}{\longrightarrow} \mathrm{H}_{n-1}\left(\mathrm{G} ; M^{\prime}\right) \longrightarrow \cdots \quad$ e 
$\cdots \stackrel{\delta}{\longrightarrow} \mathrm{H}^{n-1}\left(\mathrm{G} ; M^{\prime}\right) \stackrel{i^{*}}{\longrightarrow} \mathrm{H}^{n-1}(\mathrm{G} ; M) \stackrel{p^{*}}{\longrightarrow} \mathrm{H}^{n-1}\left(\mathrm{G} ; M^{\prime \prime}\right) \stackrel{\delta}{\longrightarrow} \mathrm{H}^{n}\left(\mathrm{G} ; M^{\prime}\right) \longrightarrow \cdots$ são exatas.

Demonstração: $[10$, III - 6.1].

\section{I.1.6 - Extensão e co-extensão de escalares}

Sejam $R$ e $S$ anéis e $\alpha: R \longrightarrow S$ um homomorfismo de anéis.

Suponhamos que $M$ seja um $S$-módulo. Existe naturalmente em $M$ uma estrutura de $R$-módulo a saber:

$$
r * m=\alpha(r) m
$$

dizemos neste caso que $M$ é um $R$-módulo por restriçāo de escalares.

Seja $M$ um $R$-módulo. Podemos ver $S$ como um $R$-módulo da seguinte forma:

$$
r * s=\alpha(r) s
$$

Podemos então construir $S \otimes_{R} M$. Afirmamos que $S \otimes_{R} M$ admite uma estrutura de $S$-módulo.

De fato: Definimos $s *\left(s^{\prime} \otimes m\right)=\left(s . s^{\prime}\right) \otimes m$. Dizemos neste caso que o $S$-módulo é obtido do $R$-módulo por extensāo de escalares $R$ e $S$.

Podemos ainda construir $\mathrm{Hom}_{R}(S, M)$. Afirmamos que $\mathrm{Hom}_{R}(S, M)$ admite uma estrutura de $S$-módulos.

De fato: Definimos $(s * f)\left(s^{\prime}\right)=f\left(s s^{\prime}\right)$. Dizemos que o $S$-módulo $H_{o m}(S, M)$ é obtido do $R$-módulo M por co-extensāo de escalares. 
Nosso interesse é aplicar estes conceitos no caso em que temos um subgrupo $S$ de G, ou seja, temos $S \hookrightarrow \mathrm{G}$ e assim $\mathbb{Z} S \stackrel{\alpha}{\longrightarrow} \mathbb{Z G}$.

Se $\mathrm{M}$ é um $\mathbb{Z} G$-módulo, denotaremos por:

1. $\operatorname{Res}_{S}^{G} M$ o $\mathbb{Z G}$-módulo obtido de $M$ por restrição de escalares via $\alpha$.

2. $\operatorname{Ind}_{S}^{G} M \circ \mathbb{Z G}$-módulo $\mathbb{Z} G \otimes_{S} M$, obtido por extensão de escalares.

3. Coind ${ }_{S}^{G} M$ o $\mathbb{Z G}$-módulo $H_{o} m_{S}(\mathbb{Z G}, M)$, obtido do $\mathbb{Z} S$-módulo $M$ por coextensão de escalares.

Lema de Shapiro: Seja $S$ um subgrupo de G e M um $\mathbb{Z} S$-módulo, então:
(i) $\mathrm{H}_{*}(S ; M)=\mathrm{H}_{*}\left(\mathrm{G} ; \operatorname{Ind}_{S}^{G} M\right)$
(ii) $\mathrm{H}^{*}(S ; M)=\mathrm{H}^{*}\left(\mathrm{G} ; \operatorname{Coind}_{S}^{G} M\right)$

Demonstração: Seja $\mathbf{P} \rightarrow \mathbb{Z}$ uma resolução projetiva de $\mathbb{Z}$ de $\mathbb{Z G}$-módulos, portanto uma resolução projetiva de $\mathbb{Z}$ de $\mathbb{Z} H$-módulos.

(i) $\mathrm{H}_{*}\left(\mathrm{G} ; \mathrm{Ind}_{S}^{G} M\right) \triangleq \mathrm{H}_{*}\left(\mathrm{G} ; \mathbb{Z} \mathrm{G} \otimes_{S} M\right) \triangleq \mathrm{H}_{*}\left(\mathrm{P} \otimes_{G}\left(\mathbb{Z G} \otimes_{S} M\right)\right)=\mathrm{H}_{*}\left(\left(\mathrm{P} \otimes_{G}\right.\right.$ $\left.\mathbb{Z} G) \otimes_{S} M\right)=\mathrm{H}_{*}\left(\mathrm{P} \otimes_{S} M\right) \triangleq \mathrm{H}_{*}(H ; M) .-$

(ii) $\mathrm{H}^{*}\left(\mathrm{G} ; \operatorname{Coind}_{S}^{G} M\right) \triangleq \mathrm{H}^{*}\left(\mathrm{G} ; \operatorname{Hom}_{S}(\mathbb{Z} \mathrm{G}, M)\right) \triangleq \mathrm{H}_{*}\left(\operatorname{Hom}_{G}\left(\mathrm{P}, H o m_{S}(\mathbb{Z G}, M)\right)=\right.$ $\mathrm{H}_{*}\left(H_{o m}(\mathbf{P}, M)\right) \triangleq \mathrm{H}^{*}(S ; M)$

Fórmula de Mackey: Sejam G um grupo, $S$ um subbrupo de G e $N$ um $\mathbb{Z} G-$ módulo, então temos $\mathbb{Z G}$ isomorfismos naturais:

(i) $\mathbb{Z}\left(\frac{G}{S}\right) \otimes \mathrm{N} \stackrel{\phi}{\longrightarrow} \operatorname{Ind}_{S}^{G} \operatorname{Res}_{S}^{G} N$

(ii) $\operatorname{Hom}\left(\mathbb{Z}\left(\frac{G}{S}\right), N\right) \stackrel{\psi}{\longrightarrow} \operatorname{Coind}_{S}^{G} \operatorname{Res}_{S}^{G} N$. onde $\mathbb{Z}\left(\frac{G}{S}\right) \otimes N$ e $\operatorname{Hom}\left(\mathbb{Z}\left(\frac{G}{S}\right), N\right)$ são vistos como $\mathbb{Z} G$-módulos com a ação diagonal descrita em I.1.2. 
Demonstração: A aplicação $\phi$ é definida por $\phi(g S \otimes n)=g \otimes g^{-1} n$, $g \in \mathrm{G}, n \in N$ e estendida por linearidade. É fácil ver que $\phi$ está bem definida e é um $\mathbb{Z G}$-homomorfismo. A inversa é dada por $g \otimes n \longmapsto g S \otimes g n$.

Já a aplicação $\psi$ é definida colocando $(\psi(f))(g)=g f\left(g^{-1} S\right), \quad \forall g \in \mathrm{G} \quad$ e $\forall f \in \operatorname{Hom}\left(\mathbb{Z}\left(\frac{G}{S}\right), N\right)$. Sua inversa $\psi^{-1}$, é definida por $\left(\psi^{-1}(h)\right)(g S)=g h\left(g^{-1}\right)$, $g \in \mathrm{G}$ e $h \in \operatorname{Hom}\left(\mathbb{Z}(\mathrm{G}), \operatorname{Res}_{S}^{G} N\right)$.

\section{I.2 - HOMOLOGIA E COHOMOLOGIA RELATIVA PARA PARES DE GRUPOS}

Daremos aqui as noções de homologia e cohomologia relativa para pares de grupos, estabelecendo ainda algumas propriedades básicas que utilizaremos nos capítulos seguintes.

\subsection{1 - Definições:}

Seja $G$ um grupo e $\mathbb{S}=\left\{S_{i}, i \in I\right\}$ uma familia de subgrupos de $\mathrm{G}$ (não necessariamente distintos); nós chamaremos (G,S) um par grupo. Se $\mathbb{S} \neq \emptyset$ nós escreveremos $\mathbb{Z}\left(\frac{G}{\mathcal{S}}\right)$ para o $\mathbb{Z G - m o ́ d u l o ~ a ̀ ~ e s q u e r d a : ~}$

$$
\underset{i \in I}{\oplus} \mathbb{Z G} \otimes_{S_{i}} \mathbb{Z}=\oplus_{i \in I} \mathbb{Z}\left(\frac{G}{S_{i}}\right)
$$

que é o grupo abeliano livre gerado pelas classes laterais $x S_{i}$, para todo $i \in I$. A $\mathbb{Z G}$-ação é a induzida pela multiplicação à esquerda.

A aumentação $\varepsilon: \mathbb{Z}\left(\frac{G}{S}\right) \longrightarrow \mathbb{Z}$ é o $\mathbb{Z G - h o m o m o r f i s m o ~ d e f i n i d o ~ p o r ~} \varepsilon\left(x S_{i}\right)=$ 1 para toda classe lateral $x S_{i}$ e todo $i \in I$. Nos denotaremos seu núcleo por $\Delta_{\frac{G}{g}}$ 
e quando não houver dúvidas sobre que módulo estamos tomando a aumentação, o denotaremos simplesmente por $\Delta$.

Para um $\mathbb{Z G}$-módulo $M$, os grupos de homologia e cohomologia relativos para o par $(G, \mathbb{S})$ com coeficientes em $M$, são definidos como segue:

Se $\mathbb{S}=\emptyset$, as homologias e cohomologias relativas do par $(G, \emptyset)$, são os grupos absolutos $\mathrm{H}_{*}(\mathrm{G} ; M)$ e $\mathrm{H}^{*}(\mathrm{G} ; M)$ respectivamente.

Se $\mathbb{S} \neq \emptyset$, colocamos para todo $k \in \mathbb{Z}:$

$\mathrm{H}_{k}(\mathrm{G}, \mathbb{S} ; M) \triangleq \mathrm{H}_{k-1}(\mathrm{G} ; \Delta \otimes M) \quad$ e $\quad \mathrm{H}^{k}(\mathrm{G}, \mathbb{S} ; M) \triangleq \mathrm{H}^{k-1}(\mathrm{G} ; H o m(\Delta, M))$, onde $\mathbb{Z G}$ atua diagonalmente em $\Delta \otimes M$ e em $\operatorname{Hom}(\Delta, M)$.

\section{I.2.2 - Resultado Básico:}

É conveniente escrever, para qualquer familia de grupos $\mathbb{S}=\left\{S_{i}, i \in I\right\}$

$$
\mathrm{H}_{k}(\mathbb{S} ; M)=\bigoplus_{i \in I} \mathrm{H}_{k}\left(S_{i} ; M\right) \quad \text { e } \quad \mathrm{H}^{k}(\mathbb{S} ; M)=\prod_{i \in I} \mathrm{H}^{k}\left(S_{i} ; M\right)
$$

aqui $M$ é um $\mathbb{Z S}$-módulo, isto é, um $\mathbb{Z} S_{i}$-módulo para todo $i \in I$.

Para um para grupo $(\mathrm{G}, \mathbb{S})$, um $\mathbb{Z} G$-módulo $M$ é um $\mathbb{Z S}$-módulo por restrição.

Teorema 1: Para um par grupo $(\mathrm{G}, \mathbb{S})$ e um $\mathbb{Z} G$-módulo $M$ temos as seguintes sequências exatas longas:

(I) $\cdots \longrightarrow \mathrm{H}^{k}(\mathrm{G} ; M) \stackrel{\text { res }^{*}}{\longrightarrow} \mathrm{H}^{k}(\mathbb{S} ; M) \stackrel{i^{*}}{\longrightarrow} \mathrm{H}^{k+1}(\mathrm{G}, \mathbb{S} ; M) \stackrel{\delta}{\longrightarrow} \mathrm{H}^{k+1}(\mathrm{G} ; M) \stackrel{\text { res* }}{\longrightarrow} \ldots$ (II) $\cdots \longrightarrow \mathrm{H}_{k+1}(\mathrm{G} ; M) \stackrel{\partial}{\longrightarrow} \mathrm{H}_{k+1}(\mathrm{G}, \mathrm{S} ; M) \stackrel{i_{*}}{\longrightarrow} \mathrm{H}_{k}(\mathrm{~S} ; M) \stackrel{\text { cor }_{\rightarrow}}{\longrightarrow} \mathrm{H}_{k}(\mathrm{G} ; M) \stackrel{\partial}{\longrightarrow} \cdots$

Demonstração: Para demonstrar (I) consideremos a sequência exata curta $\Delta \stackrel{i}{\longmapsto}$ $\mathbb{Z}\left(\frac{G}{\mathcal{S}}\right) \stackrel{\varepsilon}{\rightarrow} \mathbb{Z}$, e seja $P \rightarrow \mathbb{Z}$ uma resolução projetiva de $\mathbb{Z}$ sobre $\mathbb{Z}$ G-módulos. Então 
$\mathbb{Z}\left(\frac{G}{\mathbf{S}}\right) \otimes \mathbf{P}=\mathbf{P}^{\prime \prime}($ com a $\mathbb{Z}(G)$-ação diagonal) é isomorfa a $\mathbb{Z}(G) \otimes \mathbf{P}$ (com $\mathbb{Z}(G)$ ação à esquerda) e assim uma resolução projetiva de $\mathbb{Z}\left(\frac{G}{\mathbf{S}}\right)$. Tomando $\Delta \otimes \mathbf{P}=\mathbf{P}^{\prime}$, teremos que $\mathbf{P}^{\prime} \longrightarrow \Delta$ é uma resolução projetiva de $\Delta$. Com isso obtemos :

(i) para qualquer $\mathbb{Z} G$-módulo coeficiente $M$, a homologia destes complexos são:

$$
\begin{aligned}
& \mathrm{H}_{k}\left(\mathbf{P}^{\prime} ; M\right) \cong \mathrm{H}_{k+1}(\mathrm{G}, \mathbb{S} ; M) \\
& \mathrm{H}_{k}\left(\mathbf{P}^{\prime \prime} ; M\right) \cong \mathrm{H}_{k}(\mathbb{S} ; M) \\
& \mathrm{H}_{k}(\mathbf{P} ; M) \cong \mathrm{H}_{k+1}(\mathrm{G} ; M)
\end{aligned}
$$

analogamente para cohomologia.

(ii) o seguinte diagrama de cadeias:

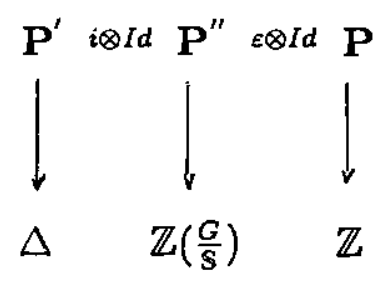

cuja sequência exata longa de cohomologia nos fornece (I) e a sequência exata longa de homologia nos fornece (II).

\section{I.3- O PRODUTO CAP ABSOLUTO E RELATIVO}

Neste parágrafo estudaremos o conceito chave para as dualidades de grupo e de pares de grupos. Como utilizaremos no desenvolvimento do trabalho, duas definições distintas para o produto cap, dependendo do que estaremos abordando. daremos aqui estas duas definições que são equivalentes.

\subsection{1- Definições e notações:}


A resolução Bar descrita em I.1.3 de um grupo G será denotada de agora em diante por $\mathrm{B}(\mathrm{G})$ e num nível $\mathrm{n}$ por $\mathrm{B}_{n}(\mathrm{G})$ ou simplesmente por $\mathrm{B}$ e $\mathrm{B}_{n}$ quando não houver dúvidas quanto ao grupo que estamos tomando a resolução Bar.

Sejam $\mathrm{C}$ e A $\mathbb{Z} G$-módulos. O produto cap: .

_ $\ldots:\left(C \otimes_{G} B_{n}\right) \otimes \operatorname{Hom}_{G}\left(B_{k}, A\right) \longrightarrow(C \otimes A) \otimes_{G} B_{n-k}$ de um elemento $e=c \otimes\left(x_{0}, \ldots, x_{n}\right) \in C \otimes_{G} B_{n}$ com $f \in \operatorname{Hom}_{G}\left(B_{k}, A\right)$ é definido como segue:

$e \frown f=\left(c \otimes\left(x_{0}, \ldots, x_{n}\right)\right) \frown f=c \otimes f\left(x_{0}, \ldots, x_{k}\right) \otimes\left(x_{k}, \ldots, x_{n}\right)$

É fácil verificar que $\partial(e \frown f)=(-1)^{k} \partial e \frown f+e \frown \delta f$, onde $k=\operatorname{deg}(f)$ (demonstraremos este resultado utilizan do a segunda definição de produto cap). Isto induz o produto cap em homologia:

- $\frown$. : $\mathrm{H}_{n}(\mathrm{G} ; C) \otimes \mathrm{H}^{k}(\mathrm{G}, A) \longrightarrow \mathrm{H}_{n-k}(\mathrm{G}, C \otimes A)$.

A segunda definição é dada para uma resolução qualquer, mas antes necessitamos de alguns resultados sobre produto tensorial de resoluções.

Dados dois grupos $G$ e $G^{\prime}$, sejam $\mathbf{P} \rightarrow \mathbb{Z}$ resolução projetiva de $\mathbb{Z}$ sobre $\mathbb{Z G}$ e $\mathbf{P}^{\prime} \rightarrow \mathbb{Z}$ resolução projetiva de $\mathbb{Z}$ sobre $\mathbb{Z} G^{\prime}$.

Temos que $\mathbf{P} \otimes \mathbf{P}^{\prime} \rightarrow \mathbb{Z} \otimes \mathbb{Z}$ é um complexo de cadeias de $\mathbb{Z G} \otimes \mathbb{Z} \mathrm{G}^{\prime}$-módulos com a ação diagonal, onde :

$$
\left(P \otimes P^{\prime}\right)_{n}=\bigoplus_{i+j=n} P_{\imath} \otimes P_{j}
$$

Além disso:

1. $\mathbb{Z} \mathrm{G} \otimes \mathbb{Z} \mathrm{G}^{\prime} \approx \mathbb{Z}\left(\mathrm{G} \times \mathrm{G}^{\prime}\right)$,

De fato: basta tomar a aplicação $g \otimes g^{\prime} \mapsto\left(g, g^{\prime}\right)$ 
2. Se $M$ é $\mathbb{Z G - p r o j e t i v o ~ e ~} N$ é $\mathbb{Z} \mathrm{G}^{\prime}$-projetivo, então $M \otimes N$ é $\mathbb{Z}\left(\mathrm{G} \times \mathrm{G}^{\prime}\right)$ projetivo. De fato: Como $M$ é $\mathbb{Z G}$-projetivo, existe um G-módulo $A$, tal que $M \oplus A=\underset{i \in I}{\oplus} \mathbb{Z} G$. Como $N$ é um $\mathbb{Z} G^{\prime}$-projetivo, existe um $\mathbb{Z} G^{\prime}$-módulo $B$, tal que $N \oplus B=\underset{j \in J}{\oplus} \mathbb{Z} G^{\prime}$. Assim:

$(M \otimes N) \oplus(A \otimes B)=(M \oplus A) \otimes(N \oplus B)=\bigoplus_{(i, j) \in I \times J} \mathbb{Z} G \otimes \mathbb{Z} G^{\prime}=\bigoplus_{(i, j) \in I \times J} \mathbb{Z}(G \times$ $\left.\mathrm{G}^{\prime}\right)$. Segue o resultado.

Concluímos de 2., que $\mathbf{P} \otimes \mathbf{P}^{\prime} \rightarrow \mathbb{Z}$ é um complexo de cadeias de $\mathbb{Z}(\mathrm{G} \times$ $\left.G^{\prime}\right)$-módulos projetivos.

Teorema 1: $\quad \mathbf{P} \otimes \mathbf{P}^{\prime} \rightarrow \mathbb{Z}$ é uma resolução projetiva de $\mathbb{Z}\left(\mathrm{G} \times \mathrm{G}^{\prime}\right)$-módulos projetivos.

Demonstração: Pelo Fórmula de Künneth dado em I.1.2, temos:

$$
\underset{p \in \mathbf{Z}}{\oplus} \mathrm{H}_{p}(\mathbf{P}) \otimes \mathrm{H}_{n-p}\left(\mathrm{P}^{\prime}\right) \mapsto \mathrm{H}_{n}\left(\mathbf{P} \otimes \mathrm{P}^{\prime}\right) \rightarrow \underset{p \in \mathbf{Z}}{\oplus} \operatorname{Tor}\left(\mathrm{H}_{p}(\mathbf{P}), \mathrm{H}_{n-p-1}\left(\mathbf{P}^{\prime}\right)\right) .
$$

Como temos duas resoluções $\mathbf{P} \rightarrow \mathbb{Z}$ e $\mathbf{P}^{\prime} \rightarrow \mathbb{Z}$ projetivas, segue que os $1^{\circ}$ e $3^{\circ}$ termos da sequência acima são nulos e portanto $\circ 2^{\circ}$ também.

Corolário: Se $\varepsilon: \mathbf{P} \rightarrow \mathbb{Z}$ e $\varepsilon^{\prime}: \mathbf{P}^{\prime} \rightarrow \mathbb{Z}$ são $\mathbb{Z G - r e s o l u c ̧ o ̃ e s ~ p r o j e t i v a s ~ d e ~} \mathbb{Z}$ sobre $\mathbb{Z} G$, então $\varepsilon \otimes \varepsilon^{\prime}: \mathbf{P} \otimes \mathbf{P}^{\prime} \rightarrow \mathbb{Z}$ também o é, onde $G$ atua diagonalmente em $\mathbf{P} \odot \mathbf{P}^{\prime}$

* Observação:

No corolário, se $\mathbf{P}=\mathbf{P}^{\prime}$, temos duas aplicações entre resoluções, a saber, $I d \otimes \varepsilon: \mathbf{P} \otimes \mathbf{P} \rightarrow \mathbf{P}$ e $\varepsilon \otimes I d: \mathbf{P} \otimes \mathbf{P} \rightarrow \mathbf{P}$. No sentido contrário, tal aplicação é denominada aproximaçāo diagonal e denotada por $\Delta: \mathbf{P} \rightarrow \mathbf{P} \otimes \mathbf{P}$. 
No caso da resolução padrão, existe uma bem conhecida aproximação diagonal chamada aplicação de Alexander-Whitney, dada por:

$$
\Delta\left(x_{0}, \ldots, x_{n}\right)=\sum_{i=0}^{n}\left(x_{0}, \ldots, x_{i}\right) \otimes\left(x_{i}, \ldots, x_{n}\right)
$$

Transladando para a resolução Bar, encontramos:

$$
\Delta\left[x_{1}|\ldots| x_{n}\right]=\sum_{i=0}^{n}\left[x_{1}|\ldots| x_{i}\right] \otimes x_{1} \ldots x_{p}\left[x_{i}|\ldots| x_{n}\right]
$$

A segunda maneira de definir o produto cap será dado a seguir.

Consideremos três $\mathbb{Z} G$-módulos $A, B$ e $C$ e suponhamos que tenhamos um pairing $\varphi: C \otimes A \longrightarrow B$. O objetivo é definir:

$$
-\frown \text {. }_{\mathrm{H}+r}(\mathrm{G} ; C) \otimes \mathrm{H}^{r}(\mathrm{G} ; A) \rightarrow \mathrm{H}_{n}(\mathrm{G} ; B) .
$$

Tomemos $e \in(\mathbf{P} \otimes \mathbf{P})_{n+r} \otimes_{G} C=\left(\sum_{i=0}^{n+r} P_{i} \otimes P_{n+r-i}\right) \otimes_{G} C$. Então temos:

$\left((\mathbf{P} \otimes \mathbf{P})_{n+r} \otimes_{G} C\right) \otimes H_{o m}\left(P_{r}, A\right) \stackrel{\frown}{\longrightarrow} P_{n} \otimes_{G} B$. Se $e=p \otimes q \otimes c$, por definição:

$$
e \frown f=q \otimes \varphi(c \otimes \overbrace{f(p)}^{(*)}) \in P_{n} \otimes_{G} B
$$

$\left.{ }^{*}\right)$ aqui entender que $f$ se anula fora da dimensão, ou seja, nesse caso $f(p)=0$ se $p \notin P_{r}$

Propriedade 1: $\partial(e \frown f)=(-1)^{|f|} \partial e \frown f+e \frown \delta f$.

Demonstração: (1) $\quad \partial(e \frown f)=\partial(q \otimes \varphi(c \otimes f(p)))=\partial q \otimes \varphi(c \otimes f(p))$

(2) $\quad \delta f=(-1)^{|f|+1} f_{o} \partial \quad$ dado em I.1.5.

$\partial e=\partial(p \otimes q \otimes c)=\partial p \otimes q \otimes c+(-1)^{|p|} p \otimes \partial q \otimes c=\partial p \otimes q \otimes c+(-1)^{|f|} p \otimes \partial q \otimes c$.

$\partial e \frown f=q \otimes \varphi(c \otimes f(\partial p))+(-1)^{|f|} \partial q \otimes \varphi(c \otimes f(p))=$

$\stackrel{(2)}{=}(-1)^{|f|+1} q \otimes \varphi(c \otimes(\delta f)(p))+(-1)^{|f|} \partial q \otimes \varphi(c \otimes f(p))=$ 
$\stackrel{(1)}{=}(-1)^{|f|+1}(p \otimes q \otimes c) \frown \delta f+(-1)^{|f|} \partial(e \frown f)$. Concluímos que:

$(-1)^{|f|}(\partial e \frown f)=(-1)(e \frown \delta f)+\partial(e \frown f)$. Segue a propriedade.

Assim temos definido o produto cap desejado. Em geral $B=C \otimes A$ e $\varphi=I d$

* Observação: Através da aproximação diagonal $\Delta$ definida acima podemos mostrar facilmente que as duas definições dadas de produto cap coincidem.

\section{I.3.2 - O produto cap relativo}

Se $\mathbb{S}$ é uma familia de subgrupos de $\mathrm{G}$, definimos:

(i) ${ }_{-} \frown_{-}: \mathrm{H}_{n+r}(\mathrm{G}, \mathbb{S} ; C) \otimes \mathrm{H}^{r}(\mathrm{G} ; A) \longrightarrow \mathrm{H}_{n}(\mathrm{G}, \mathbb{S} ; C \otimes A)$

(ii) _ _ _ : $\mathrm{H}_{n+r}(\mathrm{G}, \mathbb{S} ; C) \otimes \mathrm{H}^{r}(\mathrm{G}, \mathbb{S} ; A) \longrightarrow \mathrm{H}_{n}(\mathrm{G} ; C \otimes A)$

Para definir (i) tomamos $e=p \otimes q \otimes d \otimes c \in(\mathbf{P} \otimes \mathbf{P})_{n+r} \otimes_{G} \Delta \otimes C$ e $f \in$ $\operatorname{Hom}_{G}\left(P_{r}, A\right)$. Então:

$e \frown f \triangleq q \otimes d \otimes c \otimes f(p) \in \mathbf{P}_{n} \otimes_{G} \Delta \otimes C \otimes A$

Para definir (ii), se $e=p \otimes q \otimes d \otimes c \in(\mathbf{P} \otimes \mathbf{P})_{n+r} \otimes_{G} \Delta \otimes C$ e $f \in \operatorname{Hom}_{G}\left(P_{r}, \operatorname{Hom}(\Delta, A)\right)$. Então:

$e \frown f \triangleq q \otimes c \otimes f(p)(d) \in \mathbf{P}_{n} \otimes_{G} C \otimes A$

Em $[9, \S 2$, teorema 2.1] é demonstrado o seguinte resultado:

Teorema 1: Sejam $(\mathrm{G}, \mathbb{S})$ um par grupo com $\mathbb{S} \neq \phi, A$ e $C$ G-módulos e $e \in$ $\mathrm{H}_{n}(\mathrm{G}, \mathbb{S} ; C)$. Então o diagrama: 


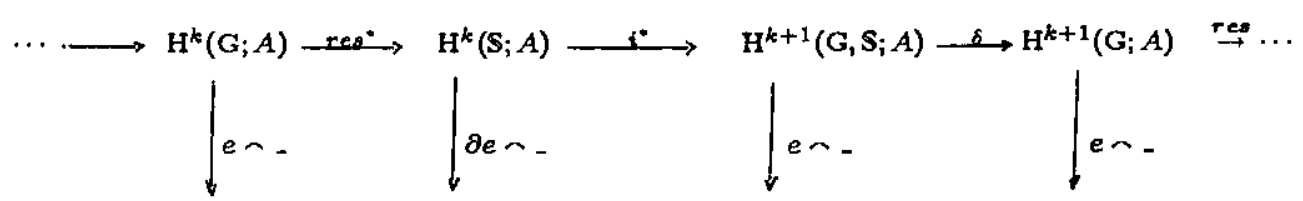

$\cdots \rightarrow \mathrm{H}_{n-k}(\mathrm{G}, \mathrm{S} ; C \otimes A) \underset{i_{0}}{\rightarrow} \mathrm{H}_{n-k-1}(\mathrm{~S} ; C \otimes A) \underset{\text { cor. }}{\overrightarrow{H_{n-k-1}}(\mathrm{G} ; C \otimes A)} \vec{\theta} \mathrm{H}_{n-k-1}(\mathrm{G}, \mathrm{S} ; C \otimes A) \overrightarrow{i_{0}} \cdots$

é comutativo a menos de sinal.

*Observação: As linhas são exatas pelo Teorema 1 de I.2.2.

\section{I.4 - GRUPOS DUALIDADES E PARES DUALIDADES}

Neste parágrafo definiremos o conceito de grupo dualidade de Poincaré, que é um grupo cuja homologia e cohomologia satisfazem relações de dualidades análogas àquelas válidas para variedades compactas.

Veremos também o conceito de pares dualidades de Poincaré cuja homologia e cohomologia satisfazem relações de dualidades similares às de variedades com bordo.

I.4.1 - Os grupos dualidades e grupos dualidades de Poincaré :

Definiçōes:

1. Um grupo $\mathrm{G}$ é denominado um grupo dualidade de dimensāo $\mathbf{n}\left(D^{n}\right.$-grupo $)$ se existe um "módulo dualizante" $\mathrm{C}$ e uma "classe fundamental" $e \in \mathrm{H}_{n}(\mathrm{G} ; C)$ tal que o produto cap com $e$ induz um isomorfismo:

$e \frown \frown_{-}: \mathrm{H}^{k}(\mathrm{G} ; A) \stackrel{\cong}{\rightrightarrows} \mathrm{H}_{n-k}(\mathrm{G} ; C \otimes A)$ para todo $k$ e todo G-módulo $A$. 
2. Se $\mathrm{G}$ é um $\mathrm{D}^{n}$-grupo e se o módulo dualizante $\mathrm{C}$ é cíclico infinito, então $\mathrm{G}$ é chamado grupo dualidade de Poincaré de dimensāo n $\left(P D^{n}\right.$-grupo $)$ e ele será orientável se a G-ação em C é trivial e nāo-orientável se a G-ação em C é não-trivial.

Proposição 1: [6, proposição 5.2.1]: Sejam G um $\mathrm{D}^{n}$-grupo sobre $K$ e $C$ seu módulo dualizante. Então as seguintes afirmações se verificam:

(i) $C \cong \mathrm{H}^{n}(\mathrm{G} ; \mathrm{KG})$ como KG-módulo à direita.

(ii) $c d_{K} \mathrm{G}=h d_{K} \mathrm{G}=n$.

(iii) $\mathrm{H}_{n}(\mathrm{G} ; C) \cong \mathrm{K}$.

(iv) para todo KG-módulo induzido $A=L \otimes_{\mathrm{K}} \mathrm{KG}$ temos $\mathrm{H}^{k}(\mathrm{G} ; A)=0$, se $k \neq n$ e um isomorfismo natural $\mathrm{H}^{n}(\mathrm{G} ; A) \stackrel{\cong}{\longrightarrow} C \otimes_{\mathrm{K}} L$.

Teorema 1: $\left[5\right.$, teorema 3.2]: Se G é um $\mathrm{D}^{n}$-grupo, assim o é qualquer subgrupo $\mathrm{S}$ de índice finito em G. A restrição res, é um isomorfismo:

$$
\mathrm{H}_{n}(\mathrm{G} ; C) \cong \mathrm{H}_{n}(S ; C)
$$

aplicando a classe fundamental de $\mathrm{G}$ na classe fundamental de $\mathrm{S}$.

Teorema 2: $[$ [5, teorema 3.5]: Seja $N \mapsto \mathrm{G} \rightarrow Q$ uma extensão de grupos. Suponhamos que $N$ e $Q$ sejam PD-grupos de dimensão $n$ e $m$ respectivamente. Então $\mathrm{G}$ é um $P D^{n+m}$-grupo e seu módulo dualizante $C_{\mathrm{G}}=\mathrm{H}^{n+m}(\mathrm{G} ; \mathbb{Z} \mathrm{G})$ é o produto tensorial dos módulos dualizantes $C_{N}=\mathrm{H}^{n}(N ; \mathbb{Z} N)$ e $C_{Q}=\mathrm{H}^{m}(Q ; \mathbb{Z} Q)$. Teorema 3: Seja $N \mapsto \mathrm{G} \rightarrow Q$ uma extensão de grupos. Suponhamos que $N$ seja um $\mathrm{PD}^{n}$-grupo e $Q$ um $\mathrm{PD}^{m}$-grupo. Nesse caso $\mathrm{G}$ é orientável $\Leftrightarrow \mathrm{N}$ é orientável e a ação diagonal de $Q$ em $C_{N}$ é trivial. 
Demonstração :

$(\Rightarrow)$ Se $Q$ não atua trivialmente em $C_{N}$, então $\left(C_{N}\right)^{Q}=\{0\}$. Assim:

$\{0\}=\left(\left(C_{N}\right)^{Q}\right)^{N} \cong\left(C_{N}\right)^{\mathrm{G}} \Rightarrow \mathrm{G}$ não é orientável.

$(\Leftarrow)$ Se $Q$ atua trivialmente em $C_{N}$, como $N$ é orientável, temos que $N$ atua trivialmente em $C_{N}$. Seja:

$\mathrm{G} \times C_{\mathrm{G}} \longrightarrow C_{\mathrm{G}}=C_{N} \otimes C_{Q}$. Vamos escrever $m \in C_{\mathrm{G}} \cong \mathbb{Z}$ como $z_{N} \otimes z_{Q}$.

Assim teremos:

$m \stackrel{(1)}{=}(g N) . m \stackrel{(2)}{=} g m$.

(1) Por hipótese $Q$ atua trivialmente em $C_{N}$ e $Q$ é orientável, assim $Q$ atua trivialmente em $C_{Q}$.

(2) Se $g^{\prime} N=g N$, então $g^{-1} g^{\prime} \in N \Rightarrow g m \stackrel{(*)}{=} g g^{-1} g^{\prime} m=g^{\prime} m$.

(*) Segue, também do fato de $Q$ ser orientável, pois neste caso, obviamente $N$ atua trivialmente em $C_{Q}$

O teorema a seguir encontra-se demonstrado em [13, teorema V.1.1].

Teorema 4: Se G $=\pi_{1}(X)$, onde $X$ é uma variedade fechada, conexa de dimensão $n$, asférica( isto é, que tem o mesmo tipo de homotopia de um complexo $K(G, 1)$ ), então $G$ é um $P D^{n}$-grupo sobre $\mathbb{Z}$.

I.4.2 - Condições de finitude 
1. Dizemos que um grupo $\mathrm{G}$ é de tipo $(\mathrm{FP})_{n}$ sobre $\mathbf{K}, n \in \mathbb{N}$, se existe uma resolução projetiva de $\mathrm{K}$ sobre $\mathrm{KG}, \mathbf{P} \rightarrow K$, com $P_{i}$ finitamente gerados (FG) com KG-módulos, para todo $i \leq n$. Se os módulos $P_{i}$ são FG para todo $i$, então dizemos que $\mathrm{G}$ é do tipo $(\mathbf{F P})_{\infty}$.

2. Um grupo $G$ é denominado grupo de tipo FP sobre $K$, se existe uma resolução KG-projetiva de $\mathrm{K}$ de comprimento finito:

$$
0 \rightarrow P_{n} \rightarrow \ldots \rightarrow P_{0} \rightarrow K \rightarrow 0
$$

*Observação:

É fácil ver que $\mathrm{G}$ é de tipo $\mathrm{FP}$ sobre $\mathrm{K}$ se, e somente se, $\mathrm{G}$ é de tipo $(\mathrm{FP})_{\infty}$ sobre $\mathrm{K}$ e $c d_{K} \mathrm{G}<\infty$.

Proposição 1 [7, proposição 2.1$]$ : $\mathrm{G}$ é de tipo $(\mathrm{FP})_{1}$ sobre $\mathrm{K}$ se, e somente se, $\mathrm{G}$ é FG.

As vezes é conveniente utilizarmos a seguinte caracterização de grupos dualidades:

Proposição 2 [7, teorema 9.2]: G é um $\mathrm{D}^{n}$-grupo sobre $\mathrm{K}$ se, e somente se, as seguintes condições ocorrem:

(i) G é de tipo FP sobre $\mathrm{K}$.

(ii) $\mathrm{H}^{k}(\mathrm{G} ; \mathrm{KG})=0$, para todo $k \neq n$.

(iii) $\mathrm{H}^{n}(\mathrm{G} ; \mathrm{KG})=C$, é flat como $\mathrm{K}$-módulo.

Corolário: Se G é um D-grupo sobre $\mathrm{K}$ então G é FG.

Demonstração: Se G é de dualidade, então pela proposição 2 , G é de tipo FP, e 
portanto de tipo $\mathrm{FP}_{1}$, pela observação acima. $\mathrm{O}$ resultado segue da proposição 1.

Teorema 1 [7, teorema 9.11]: Seja $N \hookrightarrow \mathrm{G} \rightarrow Q$ uma extensão de grupos com $c d_{k} Q<\infty$ e $N$ do tipo $(\mathrm{FP})_{\infty}$ sobre K. Se G é um grupo dualidade, então $N$ e $Q$ também o são.

\section{I.4.3 - Pares dualidades e pares dualidades de Poincaré}

Definições:

1. Um par grupo $(\mathrm{G}, \mathbb{S})$ é chamado um par dualidade de dimensāo $n$ sobre $\mathbf{K}\left(\mathrm{D}^{n}\right.$-par sobre $\left.\mathrm{K}\right)$, se existe um "módulo dualizante" $\mathrm{C}$ e uma "classe fundamental" $e \in \mathrm{H}_{n}(\mathrm{G}, \mathbb{S} ; C)$ tal que o produto cap:

$$
\begin{aligned}
& (e \frown-): \mathrm{H}^{k}(\mathrm{G} ; A) \longrightarrow \mathrm{H}_{n-k}(\mathrm{G}, \mathbb{S} ; C \otimes A) \mathrm{e} \\
& (e \frown-): \mathrm{H}^{k}(\mathrm{G}, \mathbb{S} ; A) \longrightarrow \mathrm{H}_{n-k}(\mathrm{G} ; C \otimes A)
\end{aligned}
$$

são isomorfismos para todo KG-módulo $\mathrm{A}$ e todo $k \in \mathbb{Z}$.

2. Um $D^{n}$-par $(G, \mathbb{S})$ é chamado um par dualidade de Poincaré sobre $\mathbf{K}$ $\left(\mathrm{PD}^{n}\right.$-par sobre $\left.\mathrm{K}\right)$, se seu módulo dualizante $\mathrm{C}$ é isomorfo a $\mathbb{Z}$ como um grupo abeliano.

3. No caso de $\mathrm{PD}^{n}$-par sobre $\mathrm{K}$, escrevemos $\widetilde{\mathbb{Z}}$ para $\mathrm{C}$ e $\tilde{A}$ para $\tilde{\mathbb{Z}} \otimes A$ (com ação diagonal) para qualquer KG-módulo $\mathrm{A}$. Se a $\mathrm{KG}$-ação em $\widetilde{\mathbb{Z}}$ é trivial $(\tilde{A}=A)$, o $\mathrm{PD}^{n}$-par é orientável e caso contrário nāo-orientável. 
1. Se $\mathbb{S}=\phi$, entendemos que $\mathrm{G}$ é um grupo dualidade no sentido da definição 1 de I.4.1.

2. Segue da definição 1 que se $(G, \mathbb{S})$ é um $D^{n}$-par, então $H^{n}(G, \mathbb{S} ; \mathbb{Z} G) \cong$ $\mathrm{H}_{0}(\mathrm{G}, C \otimes \mathbb{Z G}) \cong C$, como KG-módulo, assim C, é determinado pelo $\mathrm{D}^{n}$-par $(\mathrm{G}, \mathbb{S})$. Além disso $\mathrm{H}_{n}(\mathrm{G}, \mathbb{S} ; C) \cong \mathrm{H}^{0}(\mathrm{G} ; \mathbb{Z}) \cong \mathbb{Z}$ é gerado por $e$.

3. Desde que $\mathrm{H}^{k}(\mathrm{G}, \mathbb{S} ; A)=0$ para todo $\mathrm{A}$ e todo $k>n$, e $\mathrm{H}^{n}(\mathrm{G}, \mathbb{S} ; \mathbb{Z G})=C \neq 0$, temos que $c d(\mathrm{G}, \mathbb{S})=n$ e assim $n$ é determindado pelo $\mathrm{D}^{n}$-par $(\mathrm{G}, \mathbb{S})$.

O teorema a seguir encontra-se demonstrado em [9, teorema 4.2$]$.

Teorema 1: Seja $(\mathrm{G}, \mathbb{S}), \mathbb{S}=\left\{S_{i}, i \in I\right\} \neq \phi$ um $\mathrm{D}^{n}$-par com módulo dualizante C. Então:

(i) G é um grupo dualidade de dimensão $n-1$ com módulo dualizante $\Delta \otimes C$ (com $\mathbb{Z} G$-ação diagonal).

(ii) S é uma família de subgrupos finitos.

(iii) Cada $S_{i}$ é um grupo dualidade de dimensão $n-1$ com módulo dualizante $C$ (considerado como um $S_{i}$-módulo por restrição).

(iv) Uma classe fundamental $e \in \mathrm{H}_{n}(\mathrm{G}, \mathbb{S} ; C)$ determina uma classe fundamental $e_{i} \in \mathrm{H}_{n-1}\left(S_{i} ; C\right)$ para cada $S_{i}$; a saber $\partial e=\sum e_{i}$, onde $\partial$ é o homomorfismo de conexão dado no teorema 1 de I.2.2.

\section{I.5 - O PRODUTO CUP}

Nesta secção, definiremos o produto cup e algumas de suas propriedades que será ferramenta para o capítulo III. 


\section{I.5.1 - O produto cross}

Dados os grupos $G$ e $G^{\prime}$, o $\mathbb{Z G - m o ́ d u l o ~} M$ e o $\mathbb{Z G}$-módulo $M^{\prime}$, queremos definir:

$$
\mathrm{H}^{i}(\mathrm{G}, M) \otimes \mathrm{H}^{j}\left(\mathrm{G}^{\prime}, M^{\prime}\right) \rightarrow \mathrm{H}^{i+j}\left(\mathrm{G} \times \mathrm{G}^{\prime}, M \otimes M^{\prime}\right)
$$

Seja $\mathbf{P} \rightarrow \mathbb{Z}$ uma $\mathbb{Z G}$-resolução projetiva de $\mathbb{Z}$ e $\mathbf{P}^{\prime} \rightarrow \mathbb{Z}$ uma $\mathbb{Z} G^{\prime}$-resolução projetiva de $\mathbb{Z}$. Vimos no teorema 1 de I.3.1, que $\mathbf{P} \otimes \mathbf{P}^{\prime} \stackrel{\varepsilon \otimes \varepsilon^{\prime}}{\rightarrow} \mathbb{Z}$ é uma $\mathbb{Z}(\mathrm{G} \times$ $\left.G^{\prime}\right)$-resolução projetiva de $\mathbb{Z}$.

Existe um isomorfismo $\varphi: \operatorname{Hom}_{\mathrm{G}}(\mathbf{P}, M) \otimes \operatorname{Hom}_{\mathrm{G}^{\prime}}\left(\mathbf{P}^{\prime}, M\right) \rightarrow \operatorname{Hom}_{\mathrm{G} \times \mathrm{G}^{\prime}}(\mathbf{P} \otimes$ $\left.\mathbf{P}^{\prime}, M \otimes M^{\prime}\right)$, dada por:

$\varphi(u \otimes v)(\alpha \otimes \beta)=(-1)^{\operatorname{deg} v \operatorname{deg} \alpha} u(\alpha) \otimes v(\beta), \quad \forall \alpha \in \mathbf{P}, \forall \beta \in \mathbf{P}^{\prime}$

Escrevemos $u \times v=\varphi(u \otimes v)$ e é fácil verificar que $\delta(u \times v)=\delta u \times v+$ $(-1)^{p} u \times \delta v \quad$ se $\operatorname{deg} u=p$.

Isto induz a aplicação em cohomologia desejada. denominada cohomologia produto cross.

\section{I.5.2 - $\underline{\text { O produto cup }}$}

Dado $u \in \mathrm{H}^{p}(\mathrm{G}, M)$ e $v \in \mathrm{H}^{q}(\mathrm{G}, N)$, nós definimos o produto cup de $u$ e $v$ (denotado por $u \smile v$ ou $u v$ ) por um elemento $\mathrm{d}^{*}(u \times v) \in \mathrm{H}^{p+q}(\mathrm{G}, M \otimes N$ ), onde d: $\mathrm{G} \rightarrow \mathrm{G} \times \mathrm{G}$ é a aplicação diagonal. Aqui $M \otimes N$ tem a $\mathbb{Z G}$-ação diagonal de tal modo que $\mathrm{d}^{*}: \mathrm{H}^{*}(\mathrm{G} \times \mathrm{G}, M \otimes N) \rightarrow \mathrm{H}^{*}(\mathrm{G}, M \otimes N)$ faça sentido. 
Uma outra maneira de se definir o produto cup é utilizar uma $\mathbb{Z G}$-resolução projetiva $\mathbf{P} \rightarrow \mathbb{Z}$, uma aproximação diagonal $\Delta: \mathbf{P} \rightarrow \mathbf{P} \otimes \mathbf{P}$ e fazer $u \smile v=$ $(u \times v)_{\circ} \Delta \in \operatorname{Hom}_{\mathrm{G}}(\mathbf{P}, M \otimes N)$ para $u \in H_{o m}(\mathbf{P}, M)$ e $v \in H_{\mathrm{G}}\left(m_{\mathrm{G}}(\mathbf{P}, N)\right.$.

Exemplo: Com a resolução Bar definida em I.1.4 e a aplicação $\Delta$ de AlexanderWhitney dada em I.3.1, dado $u \in \operatorname{Hom}_{\mathrm{G}}\left(B_{p}, M\right)$ e $v \in \operatorname{Hom}_{\mathrm{G}}\left(B_{q}, N\right)$ :

$$
(u \smile v)\left(\mathrm{g}_{1}|\ldots| g_{p+q}\right)=(-1)^{p q} u\left(\mathrm{~g}_{1}|\ldots| g_{p}\right) \otimes g_{1} \ldots g_{p} v\left(\mathrm{~g}_{p+1}|\ldots| g_{p+q}\right) .
$$

Daremos a seguir algumas propriedades do produto cup.

Propriedade 1 (Naturalidade com respeito aos homomorfismos de grupos)[10, pag 112]: Dado $\alpha: H \rightarrow \mathrm{G}$, um homomorfismo de grupo, temos que:

$$
\alpha^{*}(u \smile v)=\alpha^{*} u \smile \alpha^{*} v \text { para qualquer } u \in \mathrm{H}^{*}(\mathrm{G}, M) \text { e } v \in \mathrm{H}^{*}(\mathrm{G}, N) .
$$

\section{Consideremos a aplicaçāo evaluaçāo:}

$$
H o m_{\mathrm{G}}(\mathbf{P}, M) \otimes \operatorname{Hom}_{\mathrm{G}}(\mathbf{P}, N) \rightarrow M \otimes_{\mathrm{G}} N \text { dado por } u \otimes(x \otimes n) \longmapsto
$$
$u(x) \otimes n$. Denotamos por $\langle u, z\rangle$ a imagem de $u \otimes z$ sob esta aplicação. Obviamente a aplicação evaluação é um aplicação de cadeia, isto é, $\langle\delta u, z\rangle+(-1)^{\operatorname{deg} u}\langle u, \partial z\rangle=$ 0 , assim existe um pairing induzido:

$$
\mathrm{H}^{p}(\mathrm{G} . M) \otimes \mathrm{H}_{p}(\mathrm{G}, N) \rightarrow M \otimes_{\mathrm{G}} N \text { também denotada por }\langle\ldots,-\rangle \text {, que }
$$
independe da escolha da resolução.

Propriedade 2[10, pag. 113]: Para qualquer $u \in \mathrm{H}^{p}\left(\mathrm{G}, M_{1}\right), v \in \mathrm{H}^{q}\left(\mathrm{G}, M_{2}\right)$ e $z \in$ $\mathrm{H}^{p+q}\left(\mathrm{G}, M_{3}\right)$, temos:

$$
\langle u \smile v, z\rangle=\langle u, v \frown z\rangle .
$$


A propriedade 3 abaixo, relaciona o produto cup com a sequência espectral de Leray-Hochschild-Serre e encontra-se demonstrada em [3, §1, proposição 1.6]. Propriedade 3: Seja $\mathrm{M}$ um $\mathbb{Z G}$-módulo. O produto cup de cadeias induz um produto cup nas sequências espectrais, ou seja, quando $r=2,3, \ldots$ teremos $E_{r}^{p, q} \otimes$ $\mathrm{E}_{r}^{s, t} \rightarrow \mathrm{E}_{r}^{p+s, q+t}$ e nos subgrupos bigraduados $\mathrm{D}^{p, q}=\mathrm{F}^{p} / \mathrm{F}^{p+1} \mathrm{H}^{p+q}(\mathrm{G}, M)$, ou seja, $\mathrm{D}^{p, q} \otimes \mathrm{D}^{s, t} \rightarrow \mathrm{D}^{p+s, q+t}$.

\section{I.6 - O GRAU DE UM HOMOMORFISMO}

Nesta secção A e B serão considerados grupos abelianos.

Temos que $\operatorname{Hom}(A, B) \neq \phi$ pois sempre $0 \in \operatorname{Hom}(A, B)$. Para $\operatorname{Aut}(A)$, sabemos um pouco mais. Todo inteiro $N$ induz um endomorfismo $N: A \rightarrow A$, tal que $N(a)=\underbrace{a+\ldots+a}_{N \text { termos }}$.

*Observações:

1. Podemos ter $N_{1} \neq N_{2}$ como inteiros e $N_{1}=N_{2}$ como endomorfismos. Por exemplo:

$$
\begin{aligned}
3: \mathbb{Z}_{5} \rightarrow \mathbb{Z}_{5} & \text { e } & 8: \mathbb{Z}_{5} \rightarrow \mathbb{Z}_{5} \\
\bar{x} \mapsto 3 \bar{x} & & \bar{x} \mapsto 8 \bar{x}
\end{aligned}
$$

2. Se $N_{1}$ e $N_{2}$ induzem o mesmo endomorfismo, então $N_{1}-N_{2}=0$ como endomorfismo.

\section{I.6.1 - Definições}

O menor inteiro positivo que induz o endomorfismo nulo é chamado o expoente de $\mathbf{A}$ que será denotado por $\exp \mathbf{A}\left(\exp \mathbb{Z}_{n}=n\right)$. Se não existe inteiro 
positivo que anula $A$, então $\exp A=0(\exp \mathbb{Z}=0)$, neste caso cada inteiro induz um homomorfismo distinto dos outros.

* Observações:

1. Se A não tem torção, então $\exp A=0$.

2. Se A tem um elemento de ordem infinita, então $\exp A=0$.

3. $\operatorname{expA} \backslash\left(N_{1}-N_{2}\right)$. De fato: se $\left(N_{1}-N_{2}\right)(x)=0$ e $\left(N_{1}-N_{2}\right)$ é o menor inteiro onde isto ocorre, então $\left(N_{1}-N_{2}\right)=(\operatorname{expA}) \mathrm{q}+r \Rightarrow r x=0$, com $r<\operatorname{expA} \therefore$ $r=0$.

4. Se h: A $\rightarrow$ B é um homomorfismo e $N \in \mathbb{Z}$, temos $N \circ \mathrm{h}=\mathrm{h} \circ N$.

Notação: $N \circ \mathrm{h}=N \mathrm{~h}$.

O menor inteiro positivo $N$, tal que $N \mathrm{~h}=0$ é chamado o expoente de $\mathbf{h}$, e denotado por exph. Se não existir tal $\mathrm{N}$, exph $=0$.

5. Se $N \mathrm{~h}=0 \Rightarrow \operatorname{exph} \backslash N$. De fato: a demonstração é idêntica à demonstração da observação 3. acima.

6. $\operatorname{exph} \backslash(\exp A \cdot \exp B)$. De fato: Basta mostrar que $m h=0$ com $m=(\exp A, \exp B)$. Mas se $m=(\exp A, \exp B)$, existem $p, q \in \mathbb{Z}$ tal que $m=p(\exp A)+q(\exp B)$. Segue que:

$$
\begin{aligned}
& \operatorname{mh}(\mathrm{a})=(\mathrm{p}(\exp \mathrm{A})+\mathrm{q}(\exp \mathrm{B})) \mathrm{h}(\mathrm{a})=\mathrm{p}(\operatorname{expA}) \mathrm{h}(\mathrm{a})+\mathrm{q}(\underbrace{\exp B) h(a)}_{=0}= \\
& \operatorname{ph}((\underbrace{\exp \mathrm{A}) \mathrm{a}}_{=\mathbf{0}})=0, \quad \forall \mathrm{a} \in \mathrm{A} . \text { Assim } \mathrm{mh}=0 .
\end{aligned}
$$

7. $\operatorname{exph}=1 \Leftrightarrow h=0$ (óbvio).

Dado $h: A \rightarrow B$ um homomorfismo, seja $\tau: B \rightarrow A$ um homomorfismo 
tal que $\mathrm{h} \circ \tau=N$, onde $N: \mathrm{B} \rightarrow \mathrm{B}$ é o endomorfismo induzido pelo $N \in \mathbb{Z}$. Chamamos $\tau$ um transfer para h e $N$ é associado a $\tau$. Analogamente, se $\tau_{\mathrm{o}} \mathrm{h}$ $=N$, então $\tau$ é um cotransfer de h e $N$ é associado ao cotransfer.

*Observações:

8. Se $\operatorname{expB} \neq 0$, podemos ter mais de um inteiro associado ao transfer $\tau$. E se $\exp A \neq 0$, podemos ter mais de um inteiro associado ao cotransfer $\tau$ (óbvio).

9. $\tau=0: \mathrm{B} \rightarrow \mathrm{A}$ é transfer e cotransfer de $\mathrm{h}: \mathrm{A} \rightarrow \mathrm{B}$ com 0 como associado. Assim o conjunto dos transfers, cotransfers e associados são sempre não vazios.

O grau e o cograu de h, que serão denotados, respectivamente por degh e cdgh, é o menor inteiro positivo associado com um transfer e um cotransfer, respectivamente. Se tal inteiro positivo não existe para nenhum dos dois, então $\operatorname{degh}=0$ e $\operatorname{cdgh}=0$.

Sejam $\sum_{i} \mathrm{~A}_{i}$ e $\sum_{i} \mathrm{~B}_{i}$ somas diretas de grupos abelianos. Dizemos que um homomorfismo $\mathrm{h}: \sum_{i} \mathrm{~A}_{i} \rightarrow \sum_{i} \mathrm{~B}_{i}$ é graduado se $\mathrm{h}=\sum_{i} \mathrm{~h}_{i}$ é tal que $\mathrm{h}_{i}: \mathrm{A}_{i} \rightarrow$ $\mathrm{B}_{i}$ são homomorfismos.

\section{I.6.2 - Resultados e exemplos}

Proposição 1: Se $N$ é um inteiro associado com um (co)transfer, então (co)grau divide $N$.

Demonstração: Sejam $N_{1}, N_{2}$ associados aos transfers $\tau_{1}, \tau_{2}$, respectivamente. Então a combinação linear a $N_{1}+\mathrm{b} N_{2}$, onde $\mathrm{a}, \mathrm{b} \in \mathbb{Z}$ é um inteiro associado ao transfer $a \tau_{1}+\mathrm{b} \tau_{2}$. De fato: $\mathrm{h}_{\mathrm{o}}\left(\mathrm{a} \tau_{1}+\mathrm{b} \tau_{2}\right)=\mathrm{h}_{\circ} \mathrm{a} \tau_{1}+\mathrm{h}_{\circ} \mathrm{b} \tau_{2}=\mathrm{ah}_{\circ} \tau_{1}+\mathrm{bh} \mathrm{h}_{\mathrm{o}} \tau_{2}=$ 
$\mathrm{a} N_{1}+\mathrm{b} N_{2}$.

Portanto o conjunto dos associados é um ideal de $\mathbb{Z}$ e assim principal com gerador degh. Logo o resultado fica demonstrado para transfer. Para cotransfer a demonstração é análoga.

*Observação:

1. $\operatorname{degh} \backslash \operatorname{expB}$ e cdgh \expA. Segue imediatamente da proposição 1.

\section{Exemplo de cálculo de degh e cdgh}

Seja $h: \mathbb{Z}_{3} \rightarrow \mathbb{Z}_{6}$, tal que $h(\overline{1})=\overline{2}$. Assim $\tau: \mathbb{Z}_{6} \rightarrow \mathbb{Z}_{3}$.

Pode $h_{\circ} \tau=1$ ? Ou seja, pode $h_{\diamond} \tau(\overline{1})=\overline{1}$ ? Como h não atinge $\overline{1}$, isto é impossível.

Pode $h_{\circ} \tau=2$ ? Ou seja, pode $h_{\circ} \tau(\overline{1})=\overline{2}$ ? Tomamos $\tau(\overline{1})=\overline{1} \Rightarrow h_{\circ} \tau(\overline{1})=$ $\mathrm{h}(\overline{\mathrm{l}})=\overline{2}$. Portanto degh $=2$.

Pode $-\mathrm{oh}=1$ ? Ou seja, pode $\tau_{\mathrm{oh}}(\overline{1})=\overline{1}$ ? Tomamos $\tau(\overline{1})=\overline{2} \Rightarrow \tau_{\mathrm{oh}}(\overline{1})=$ $\tau(\overline{2})=\tau(\overline{1})+\tau(\overline{1})=\overline{2}+\overline{2}=\overline{1}$. Portanto cdgh $=1$.

* Observaçöes:

2. Grau e cograu caracterizam isomorfismos da seguinte forma:

$$
\operatorname{degh}=\operatorname{cdgh}=1 \Leftrightarrow h \text { é um isomorfismo. }
$$

De fato: $(\Rightarrow) \operatorname{degh}=1 \Rightarrow \exists \tau_{1}$ tal que $\mathrm{h}_{\mathrm{o}} \tau_{1}=\operatorname{Id}_{\mathrm{B}} \therefore \mathrm{h}$ é sobrejetora.

$$
\text { cdgh }=1 \Rightarrow \exists \tau_{2} \text { tal que } \tau_{2} \mathrm{~h}=\operatorname{Id}_{\mathrm{A}} \therefore \mathrm{h} \text { é injetora. E assim } \mathrm{h} \text { é }
$$

um isomorfismo.

$$
(\Leftarrow i \text { úbrio. }
$$


3. $\operatorname{degh} \neq 1$ e cdgh $\neq 1$ não caracterizam sobrejetividade e injetividade.

De fato: (a) Tomamos $h: \mathbb{Z} \rightarrow \mathbb{Z}_{n}$ o homomorfismo quociente. Seja $\tau \in$ $\operatorname{Hom}\left(\mathbb{Z}_{n}, \mathbb{Z}\right) . \quad$ Assim $\tau(\overline{0})=0=\tau(\bar{n})=\tau(\mathrm{n} . \overline{1})=\tau(\overline{1})+\ldots+\tau(\overline{1})=\mathrm{n} \tau(\overline{1})$. Logo $\tau(\overline{1})=0 \therefore \operatorname{Hom}\left(\mathbb{Z}_{n}, \mathbb{Z}\right)=0$ e $\operatorname{degh}=0 \neq 1$. .

(b) Tomamos $\mathrm{h}: \mathbb{Z} \rightarrow \mathbb{Z}$ tal que $\mathrm{h}(1)=\mathrm{n}$. Assim $\tau_{\mathrm{o}} \mathrm{h}(1)=\tau(\mathrm{n}) \neq 1 \therefore$ $\operatorname{cdgh} \neq 1$.

Proposição 2 : $\operatorname{degh} 2 \backslash \operatorname{deg}\left(\mathrm{h}_{2} \circ \mathrm{h}_{1}\right) \backslash\left(\operatorname{degh}_{1}\right)\left(\operatorname{degh}_{2}\right)$

Demonstração: Sejam $h_{1}: A \rightarrow B ; h_{2}: B \rightarrow C ; \underbrace{h_{2 \circ} h_{1}}_{=h_{3}}: A \rightarrow C$

Sejam $\tau_{1}: \mathrm{B} \rightarrow \mathrm{A} ; \tau_{2}: \mathrm{C} \rightarrow \mathrm{B}$ e $\tau_{3}: \mathrm{C} \rightarrow \mathrm{A}$ os transfers associados com os homomorfismos $h_{1}, h_{2}$ e $h_{3}$, respectivamente. Temos que:

$$
\mathrm{h}_{2} \circ\left(\mathrm{h}_{1} \circ \tau_{3}\right)=\mathrm{h}_{3} \circ \tau_{3}=\operatorname{degh}_{3}
$$

Assim $h_{1} \circ \tau_{3}: C \rightarrow$ B é um transfer para $h_{2}$ com degh $_{3}$ como associado. Logo $\operatorname{degh} h_{2} \backslash \operatorname{degh} h_{3}=\operatorname{deg}\left(h_{2} \circ h_{1}\right)$ pela proposição 1. Temos ainda que:

$$
\left.\mathrm{h}_{3} \circ\left(\tau_{1} \circ \tau_{2}\right)=\mathrm{h}_{2} \circ\left(\mathrm{h}_{1} \circ \tau_{1}\right) \circ \tau_{2}=\mathrm{h}_{2} \circ \operatorname{degh} \mathrm{h}_{1} \tau_{2}=\left(\operatorname{degh}_{1}\right)(\operatorname{degh})_{2}\right) .
$$

Portanto $\tau_{1} \tau_{2}$ é um tranfer para $h_{3}$ com $\left(\operatorname{degh} h_{1}\right)\left(\operatorname{degh} h_{2}\right)$ como associado. Logo pela proposição $1, \operatorname{deg}\left(h_{2} \circ h_{1}\right)=\operatorname{degh} h_{3} \backslash\left(\operatorname{degh} h_{1}\right)\left(\operatorname{degh} h_{2}\right)$.

Proposição 3: $\operatorname{cdgh}_{1} \backslash \mathrm{cdg}\left(\mathrm{h}_{2} \circ \mathrm{h}_{1}\right) \backslash\left(\mathrm{cdgh}_{1}\right)\left(\mathrm{cdgh}_{2}\right)$

Demonstração: Análoga à demonstração da proposição 2ゅ.

Proposição 4 $:$ Seja h $: \sum_{i} \mathrm{~A}_{i} \rightarrow \sum_{i} \mathrm{~B}_{i}$ um homomorfismo graduado. Então degh $=m \underset{i}{ } c\left\{\operatorname{degh}_{i}\right\}$ e $c d g h=m \underset{i}{m}\left\{\operatorname{cdgh}_{i}\right\}$. 
Demonstração: Seja $\tau: \sum_{i} \mathrm{~B}_{i} \rightarrow \sum_{i} \mathrm{~A}_{i}$ um transfer que realiza o degh, ou seja, $h \circ \tau=$ degh.

Definimos $\tau_{i}: \mathrm{B}_{i} \rightarrow \mathrm{A}_{i}$ como a composta:

$$
\mathrm{B}_{i} \stackrel{i}{\rightarrow} \sum_{i} \mathrm{~B}_{i} \stackrel{\tau}{\rightarrow} \sum_{i} \mathrm{~A}_{i} \stackrel{\pi_{i}}{\rightarrow} \mathrm{A}_{i}
$$

Afrrmamos que $\sum_{i} \tau_{i}$ é um transfer que realiza degh.

De fato: $\tau\left(b_{i}\right)=\tau\left(b_{i}\right)+x$, onde $b_{i} \in \mathrm{B}_{i}$ e $x \in \sum_{j \neq i} \mathrm{~A}_{j}$.

$$
\left.(\operatorname{degh})\left(b_{i}\right)=h \circ \tau\left(b_{i}\right)=h(\underbrace{\tau l_{i}\left(b_{i}\right)}_{\in A_{i}}+x)\right)=h_{i} \tau_{i}\left(b_{i}\right)+h(x) \text {, onde } h(x) \text { está }
$$

em $\sum_{j \neq i} \mathrm{~B}_{j}$, pois h é graduado. Logo $(\operatorname{degh})\left(b_{i}\right)=\mathrm{h}_{i} \tau_{i}\left(b_{i}\right)$ e $\mathrm{h}(x)=0$.

Assim $\left(\mathrm{h} \circ \sum_{i} \tau_{i}\right)\left(b_{1}, b_{2}, \ldots\right)=\left(\mathrm{h} \circ \tau_{1}\left(b_{1}\right), \mathrm{h} \circ \tau_{2}\left(b_{2}\right), \ldots\right)=\left((\operatorname{degh}) b_{1},(\operatorname{degh}) b_{2}, \ldots\right)$ $=\operatorname{degh}\left(b_{1}, b_{2}, \ldots\right)$.

Seja $K_{i}=\frac{m m c\left\{\operatorname{deg} h_{i}\right\}}{\operatorname{deg} h_{i}}$. Seja $\overline{\tau_{i}}$ o transfer que realiza $m_{i} m c\left\{\operatorname{deg} h_{i}\right\}$. Assim:

$$
h_{i} \circ \overline{T_{i}}=\left(\operatorname{degh} h_{i}\right) \cdot K_{i} \text { e portanto } h_{\circ} \sum_{i} \overline{\tau_{i}}=m m c\left\{\operatorname{deg} h_{i}\right\} \text {. }
$$

Logo degh $\backslash \underset{i}{m m c}\left\{\operatorname{deg} h_{i}\right\}$. Mas como $h_{i} \circ \tau_{i}=\operatorname{degh}, \forall i$, temos que $m_{i} m c$ $\left\{\operatorname{deg} h_{i}\right\} \backslash \operatorname{degh}$

* Observação:

4. Para aplicações não graduadas, o resultado não é verdadeiro. Se A e B são F.G., então $A \cong F \oplus T$ e $B \cong F^{\prime} \oplus T^{\prime}$, onde $F, F^{\prime}$ são grupos abelianos livres e $T$, $T^{\prime}$ subgrupos de torção. Um homomorfismo $h: A \rightarrow B$ não respeita a estrutura de soma direta. Exemplo:

Seja $A=\mathbb{Z} \oplus \mathbb{Z}_{8}$. Denotemos por $a$ o gerador de $\mathbb{Z}$ e por $\alpha$ o gerador de $\mathbb{Z}_{8}$. Seja $h: A \rightarrow$ A definido por $h(a)=2 a+\alpha$ e $h(\alpha)=4 \alpha$.

Afirmamos que degh $=8$. 
De fato: $\mathrm{A} \stackrel{\tau}{\rightarrow} \mathrm{A} \stackrel{\text { h }}{\rightarrow} \mathrm{A} . \mathrm{Se}(\mathrm{h} \circ \tau)(a+\alpha)=8(a+\alpha)=8 a+8 \alpha=4(2 a+\alpha)+4 \alpha$ $=4 \mathrm{~h}(a)+\mathrm{h}(\alpha)=\mathrm{h}(4 a+\alpha)$.

Assim se tomarmos $\tau(a+\alpha)=4 a+\alpha$, temos 8 associado a $\tau$ e portanto $\operatorname{degh} \backslash 8$. Assim $\operatorname{degh}=1,2,4$ ou 8.

Se degh $=4 \Rightarrow(h \circ \tau)(a+\alpha)=h(m a+n \alpha)=m(2 a+\alpha)+n(4 \alpha)=$ $2 \mathrm{~m} a+(\mathrm{m}+4 \mathrm{n}) \alpha=4(a+\alpha) \Rightarrow\left\{\begin{array}{l}2 \mathrm{~m}=4 \Rightarrow \mathrm{m}=2 \\ \mathrm{~m}+4 \mathrm{n}=4\end{array} \quad \therefore\right.$ n não é inteiro (absurdo).

Idem para 2 e 1 . Logo degh $=8$.

Por outro lado $h_{T}=h_{\mathbb{Z}_{8}}: \mathbb{Z}_{8} \rightarrow \mathbb{Z}_{8}$ é dado por $h_{T}(\alpha)=4 \alpha$.

$$
\mathbb{Z}_{8} \stackrel{\tau}{\rightarrow} \mathbb{Z}_{8} \stackrel{h_{T}}{\rightarrow} \mathbb{Z}_{8} \text {, onde }\left(\mathrm{h}_{T^{\circ}} \tau\right)(\alpha)=\mathrm{h}_{T}(\mathrm{~m} \alpha)=4 \mathrm{~m} \alpha
$$

Tomando $\mathrm{m}=1$, temos $\operatorname{degh}_{T}=4$.

Seja $h_{F}$ a composta:

$$
\mathbb{Z} \stackrel{i}{\rightarrow} \mathbb{Z} \oplus \mathbb{Z}_{8} \stackrel{h}{\rightarrow} \mathbb{Z} \oplus \mathbb{Z}_{8} \stackrel{\pi}{\rightarrow} \mathbb{Z} . \mathrm{h}_{F}(a)=2 a \therefore\left(\mathrm{h}_{F} \mathrm{o} \tau\right)(a)=\mathrm{h}_{F}(\mathrm{~m} a)=\mathrm{m} 2 a .
$$

Tomando $\mathrm{m}=1$, teremos $\operatorname{degh}_{F}=2$.

Assim degh $=8 \neq 4=\operatorname{mmc}\left\{\operatorname{degh}_{T}, \operatorname{degh}\right\}$

\section{I.7 - O ÍNDICE DE UMA EXTENSÃO DE GRUPOS}

Enunciaremos agora a versão para Grupos de Dualidade do Teorema B dado no capítulo 0.

Teorema $B^{\prime}:$ Seja $N \mapsto \mathrm{G} \rightarrow Q$ uma extensão de grupos, com $N$ um $\mathrm{PD}^{n_{-}}$ grupo, $Q$ um $\mathrm{PD}^{m}$-grupo, ambos orientáveis. Suponhamos que $Q$ atue trivial- 
mente em $\mathrm{H}^{*}(N)$. Então o índice de G é o produto dos índices de $N$ e $Q$, isto é:

$$
\sigma(\mathrm{G})=\sigma(N) \sigma(Q)
$$

Como foi dito no capítulo 0 , daremos a seguir um exemplo que mostra a necessidade de $Q$ atuar trivialmente em $\mathrm{H}^{*}(N)$.

Os artigos [2] e [21] de M.F.Atiyah e K.Kodaira, respectivamente, exibem exemplos que trabalhados de forma conveniente, mostram a necessidade de $Q$ atuar trivialmente na cohomologia de $N$, para que o Teorema $\mathrm{B}^{\prime}$ seja verificado.

$\mathrm{Na}$ verdade, nestes artigos se constroi uma fibração cujo espaço total são 4-variedades $M_{n, m}$ que possuem índice diferente de zero; como a base $S$ e a fibra F obviamente possuem dimensão dois, o produto dos índices da base e da fibra é nulo e assim após adaptações para grupos dualidades obtemos o exemplo desejado.

$$
\text { Como: } \begin{aligned}
\sigma(\mathrm{G} \times \mathrm{G}) & =\sigma(\mathrm{G}) \sigma(\mathrm{G}) \\
\sigma(N \times N) & =\sigma(N) \sigma(N) \\
\sigma(Q \times Q) & =\sigma(Q) \sigma(Q)
\end{aligned}
$$

temos exemplos para dimensões com base e fibra divisível por 4.

Lema 1: Se $\mathrm{MI}^{2}$ é compacta distinta de $\mathrm{S}^{2}$ e de $\mathbb{R} \mathrm{P}^{2}$, então $\mathrm{M}^{2} \cong K\left(\pi_{1}(M), 1\right)$.

Demonstração: Se $M \neq S^{2}, \mathbb{R} P(2)$, então $\widetilde{M}$ é uma variedade bidimensional, não compacta e $\mathrm{H}_{2}(\widetilde{M})=0$. Como $\pi_{1}(\widetilde{M})=0$, por Hurewicz, temos que o primeiro $\pi_{i}$ e o primeiro $H_{i}$ distintos de zero são isomorfos. 
Como $\pi_{2}(\widetilde{M})=0 \Rightarrow \mathrm{H}_{i}(\widetilde{M})=0$, para $i \geq 2$, pois $\operatorname{dim} \widetilde{M}=2$ e $\widetilde{M}$ é não compacta; assim sendo $\pi_{i}(\widetilde{M})=0$, para $i>0$.

Assim $\widetilde{M}$ é contrátil e $M=K\left(\pi_{1}(M), 1\right)$, por definição.

A base $\mathrm{S}$ da fibração dos artigos citados é um recobrimento não ramificado de $\mathrm{m}^{2 g}$-folhas $(\mathrm{m} \geq 2)$ sobre uma superfície orientável compacta, desta forma $\mathrm{S}$ é uma variedade compacta diferente de $S^{2}$ e de $\mathbb{R P}^{2}$. A fibra $\mathrm{F}$ dessas fibrações é uma superfície de Riemann compacta que é um recobrimento cíclico ramificado em dois pontos com $m$ folhas e assim, também não é $S^{2}$ nem $\mathbb{R P}^{2}$. Logo pelo lema 1 , temos que $S$ e $F$ são $K\left(\pi_{1}(S), 1\right)$ e $K\left(\pi_{1}(F), 1\right)^{\circ}$, respectivamente.

Aplicando a sequência longa de homotopia da fibração $F \leftrightarrow M_{n, m} \rightarrow S$ obtemos:

$\cdots \rightarrow \pi_{n}(\mathrm{~F}) \stackrel{i}{\rightarrow} \pi_{n}\left(\mathrm{M}_{n, m}\right) \stackrel{p}{\rightarrow} \pi_{n}(\mathrm{~S}) \stackrel{\delta}{\rightarrow} \pi_{n-1}(\mathrm{~F}) \rightarrow \cdots \stackrel{i}{\rightarrow} \pi_{0}\left(\mathrm{M}_{n, m}\right) \stackrel{p}{\rightarrow} \pi_{0}(\mathrm{~S})$.

Pelas observações acima $\pi_{i}(F)=\pi_{i}(\mathrm{~S})=0$, se $i>1$. Logo $\mathrm{M}_{n, m}$ é asférica e assim pelo teorema 3 de I.4.1, teremos que $\pi_{1}\left(\mathrm{M}_{n, m}\right)$ é um $\mathrm{PD}^{4}$-Grupo sobre $\mathbb{Z}$.

Pelo teorema 1 de I.1.5 obtemos o exemplo desejado.

* Observação: Para uma demonstração detalhada do teorema B, dado no capítulo 0 ver [23]. 


\section{CAPÍTULO II}

\section{UMA FÓRMULA PARA EXTENSÃO DE GRUPOS}

Em [18], Gottlieb demonstrou que para uma variedade fibrada $F \stackrel{i}{\rightarrow} E \stackrel{p}{\rightarrow} B$ a seguinte fórmula é verificada:

$i_{*}([F])=\left(p^{*}[\bar{B}]\right) \frown[E]$, onde $[E] \in \mathrm{H}_{n+m}(E),[F] \in \mathrm{H}_{n}(F)$ são classes fundamentais e $[\bar{B}]$ a classe fundamental dual em $\mathrm{H}^{m}(B)$.

Neste capítulo demonstraremos uma fórmula análoga para extensão de grupos, dada no teorema abaixo, que denominaremos Teorema principal.

Teorema principal : Seja $N \stackrel{i}{\mapsto} \mathrm{G} \stackrel{\pi}{\rightarrow} Q$ uma extensão de grupos. Suponhamos que $N$ seja um $\mathrm{PD}^{n}$-Grupo e $Q$ um $\mathrm{PD}^{m}$-Grupo ambos orientáveis, então:

$i_{*}\left(e_{N}\right)=e_{G} \frown \pi^{*} \overline{e_{Q}}$, onde $e_{G} \in \mathrm{H}_{n+m}(\mathrm{G}), e_{N} \in \mathrm{H}_{n}(N)$ e $\overline{e_{Q}} \in \mathrm{H}^{m}(Q)$ são classes fundamentais.

Observações:

1. A fórmula faz sentido quando tomamos $\pi^{*}\left(\overline{e_{Q}}\right) \in H^{m}(G)$, isto é, com coeficientes em $\mathbb{Z}$.

De fato: $i: . \mathrm{V} \rightarrow \mathrm{G}$, induz $i_{*}: \mathrm{H}_{n}(N) \rightarrow \mathrm{H}_{n}(\mathrm{G})$, ou seja, $i_{*}\left(e_{N}\right) \in \mathrm{H}_{n}(\mathrm{G})$. Por outro lado. $\epsilon_{G} \frown$ - $: \mathrm{H}^{k}(\mathrm{G}) \rightarrow \mathrm{H}_{n+m-k}(\mathrm{G})$, assim, se $k=m \Rightarrow$ 
$e_{G} \frown \frown_{-}: \mathrm{H}^{m}(\mathrm{G}) \rightarrow \mathrm{H}_{n}(\mathrm{G}) \therefore e_{G} \frown \pi^{*}\left(\overline{e_{Q}}\right) \in \mathrm{H}_{n}(\mathrm{G})$.

2. Uma classe fundamental $\overline{e_{Q}} \in \mathrm{H}^{m}(Q)$ para um $\mathrm{PD}^{m}$-Grupo $Q$ é definida como segue:

Seja $e_{Q}$ uma classe fundamental em $\mathrm{H}_{m}(Q)$ (lembrar que estamos num $\mathrm{PD}^{m_{-}}$ Grupo, assim o módulo dualizante $C_{Q}$ é $\mathbb{Z}$, e como é orientável, a $Q$-ação é trivial).

Temos que $e_{Q} \frown-: \mathrm{H}^{m}(Q) \rightarrow \mathrm{H}_{0}(Q) \cong \mathbb{Z}_{Q} \cong \mathbb{Z}$ é um isomorfismo, assim tomamos $\overline{e_{Q}}$ como sendo o elemento de $H^{m}(Q)$ tal que $e_{Q} \frown \overline{e_{Q}}=1$, ou seja, $\overline{e_{Q}}=\left(e_{Q} \frown{ }_{-}\right)^{-1}(1)$.

A idéia da demonstração é considerar primeiramente a aplicação:

$\alpha_{*} \varkappa_{*}: \mathrm{H}_{m}\left(Q ; \mathrm{H}^{m}(Q ; \mathbb{Z} Q)\right) \rightarrow \mathrm{H}_{m}\left(Q ; \mathrm{H}_{n}\left(N ; \mathrm{H}^{m}(Q ; \mathbb{Z} Q) \otimes \mathrm{H}^{n}(N ; \mathbb{Z} N)\right)\right)$ construída em [6] no teorema 2.7.1 (reproduziremos esta demonstração no teorema 1 de II.2, já que é nela que baseamos a demonstração do Teorema principal). Neste teorema, mostra-se que $\alpha_{*} \varkappa_{*}\left(e_{Q}\right)=e$ é uma classe fundamental em $\mathrm{H}_{m}\left(Q ; \mathrm{H}_{n}(N ; C)\right)$ onde $C$ é o módulo dualizante de $G$.

Assim podemos fazer $e \frown \frown_{-}: \mathrm{H}^{m}(Q) \rightarrow \mathrm{H}_{0}\left(Q ; \mathrm{H}_{n}\left(N ; \mathrm{H}^{m}(Q ; \mathbb{Z} Q) \otimes \mathrm{H}^{n}(N ; \mathbb{Z} N)\right)\right)$.

Na secção II.1 mostra-se que o produto cap (a menos de sinal) é compatível com a sequência espectral de Lyndon-Hochschild-Serre, que fornece um isomorfismo $\xi: \mathrm{H}_{m}\left(Q ; \mathrm{H}_{n}(N ; C)\right) \stackrel{\sim}{\rightarrow} \mathrm{H}_{m+n}(G ; C)$ e assim teremos, no nosso caso, que $\xi(e)$ é uma classe fundamental em $\mathrm{H}_{m+n}(\mathrm{G})$.

Utilizaremos esta classe $\xi(e)$ a nível de cadeia, para demonstrar o Teorema Principal que será feito em II.3. 
Através deste teorema, demonstraremos resultados envolvendo grau e cograu de aplicações e número de uma extensão, que serão definidos no capítulo III.

\section{II.1 - AS SEQUENNCIAS ESPECTRAIS DE LHS E A COMPATIBILI- DADE COM O PRODUTO CAP}

Este parágrafo foi extraído de $[4, \S 1,3]$.

As sequências espectrais de Lyndon-Hochschild-Serre (LHS), são sequências espectrais dos complexos bigraduados:

1. $\mathrm{K}^{p, q}(A)=\operatorname{Hom}_{G}\left(B_{p}(Q) \otimes B_{q}(\mathrm{G}), A\right) \cong \operatorname{Hom}_{Q}\left(B_{p}(Q), \operatorname{Hom}_{N}\left(B_{q}(\mathrm{G}), A\right)\right) \mathrm{com}$ as diferenciações parciais:

$$
\begin{aligned}
& \left(\delta^{\prime} f\right)\left(b^{\prime} \otimes b^{\prime \prime}\right)=(-1)^{p+q+1} f\left(d b^{\prime} \otimes b^{\prime \prime}\right), \quad f \in K^{p, q} ; b^{\prime} \in B_{p+1} \text { e } b^{\prime \prime} \in B_{q} . \\
& \left(\delta^{\prime \prime} f\right)\left(b^{\prime} \otimes b^{\prime \prime}\right)=(-1)^{q+1} f\left(b^{\prime} \otimes d b^{\prime \prime}\right), \quad f \in K^{p, q} ; b^{\prime} \in B_{p} \text { e } b^{\prime \prime} \in B_{q+1} .
\end{aligned}
$$

2. $\mathrm{K}_{s, r}(A)=A \otimes_{G}\left(B_{s}(\mathrm{G}) \otimes B_{r}(Q)\right) \cong\left(A \otimes_{N} B_{s}(\mathrm{G})\right) \otimes_{Q} B_{r}(Q)$, com as diferenciações parciais:

$$
\left.\begin{array}{c}
\partial^{\prime}\left(a \otimes b^{\prime} \otimes b^{\prime \prime}\right)=a \otimes d b^{\prime} \otimes b^{\prime \prime} \\
\left(a \otimes b^{\prime} \otimes b^{\prime \prime}\right)=(-1)^{s} a \otimes b^{\prime} \otimes d b^{\prime \prime}
\end{array}\right\} \quad a \in A, b^{\prime} \in B_{s} \text { e } b^{\prime \prime} \in B_{r}
$$

$\mathrm{K}^{*}$ e $\mathrm{K}_{*}$ são funtores da categoria dos G-módulos na categoria dos bicomplexos. Para dois G-módulos $A$ e $C$ definimos um produto cap no bicomplexo:

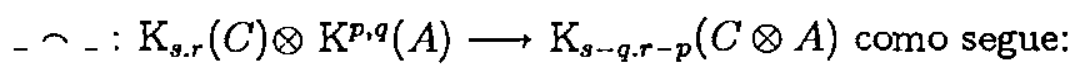

Para os elementos $e=c \otimes\left(x_{0}, \ldots, x_{s}\right) \otimes\left(y_{0}, \ldots, y_{r}\right) \in K_{s, r}(C)$ e $f \in K^{p, q}(A)$ seja

$$
e \frown f=(-1)^{s p}\left(c \otimes f\left(\left(y_{0}, \ldots, y_{p}\right) \otimes\left(x_{0}, \ldots, x_{q}\right)\right)\right) \otimes\left(x_{q}, \ldots, x_{s}\right) \otimes\left(y_{p}, \ldots, y_{r}\right)
$$


Vemos facilmente que as seguintes fórmulas são válidas:

$$
\left.\begin{array}{l}
\partial^{\prime \prime}(e \frown f)=(-1)^{p+q} \partial^{\prime} e \frown f+e \frown \delta^{\prime \prime} f \\
\partial^{\prime}(e \frown f)=(-1)^{p+q} \partial^{\prime \prime} e \frown f+e \frown \delta^{\prime} f
\end{array}\right\}
$$

“ $\frown$ " induz nos complexos totais Tot $\mathrm{K}^{*} \mathrm{e}$ Tot $\mathrm{K}_{*}$ o produto:

$-\frown_{-}:\left(\operatorname{Tot} \mathrm{K}_{*}(C)\right)_{n} \otimes\left(\operatorname{Tot} \mathrm{K}^{*}(A)\right)^{k} \longrightarrow\left(\operatorname{Tot} \mathrm{K}_{*}(C \otimes A)\right)_{n-k}$

e de $(*)$ segue que para as diferenciais totais $\delta=\delta^{\prime}+\delta^{\prime \prime}$ e $\partial=\partial^{\prime}+\partial^{\prime \prime}$ :

$$
\partial(e \frown f)=(-1)^{k} \partial e \frown f+e \frown \delta f
$$

Isto também induz um produto nas homologias dos complexos totais.

A homologia do complexo total é porém isomorfa a homologia de G. Estes isomorfismos são induzidos pelas seguintes tranformações de cadeias:

$$
\begin{gathered}
\xi: \operatorname{Hom}_{G}(B(\mathrm{G}), A) \stackrel{\xi^{\prime}}{\longrightarrow} \mathrm{K}^{0, *}(A) \hookrightarrow \operatorname{Tot} \mathrm{K}^{*}(A) \\
\eta: \operatorname{Tot} \mathrm{K}_{*}(C) \rightarrow \mathrm{K}_{*, 0}(C) \underset{\eta^{\prime}}{\longrightarrow} C \otimes \otimes_{G} B(\mathrm{G}) \text {. Aqui } \\
\left(\xi^{\prime} f\right)\left(b^{\prime} \otimes b^{\prime \prime}\right)=f\left(b^{\prime \prime}\right), \text { onde } f \in \operatorname{Hom}_{G}(B(\mathrm{G}), A), b^{\prime} \in B_{0}(\mathrm{G}) \text { e } b^{\prime \prime} \in B(\mathrm{G}) \\
\eta^{\prime}\left(c \otimes\left(b^{\prime} \otimes b^{\prime \prime}\right)\right)=c \otimes b^{\prime}, \text { onde } c \in C, b^{\prime} \in B(\mathrm{G}) \text { e } b^{\prime \prime} \in B_{0}(\mathrm{G}) .
\end{gathered}
$$

Agora é fácil mostrar que:

Lema 1: O produto cap definido sobre os bicomplexos $\mathrm{K}^{*}$ e $\mathrm{K}$, coincide com o produto cap usual na homologia de G, isto é, o seguinte diagrama é comutativo:

$$
\begin{aligned}
& \mathrm{H}_{n}\left(\operatorname{TotK}_{*}(C)\right) \stackrel{\frown}{\longrightarrow} \operatorname{Hom}\left(\mathrm{H}^{k}\left(\operatorname{TotK}^{*}(A)\right), \mathrm{H}_{n-k}\left(\operatorname{Tot} \mathrm{K}_{*}(C \otimes A)\right)\right. \\
& \eta_{*} \downarrow \quad \operatorname{Hom}\left(\xi^{*}, \eta_{*}\right) \downarrow \\
& \mathrm{H}_{n}(\mathrm{G} ; C) \stackrel{\frown}{\longrightarrow} \operatorname{Hom}\left(\mathrm{H}^{k}(\mathrm{G} ; A), \mathrm{H}_{n-k}(\mathrm{G} ; C \otimes A)\right)
\end{aligned}
$$


As duas sequências espectrais produzidas pelos complexos bigraduados $\mathrm{K}^{*} \mathrm{e}$ $\mathrm{K}_{*}$ convergem para a homologia do complexo total.

$$
E_{r+s} \Rightarrow \mathrm{H}_{r+s}\left(\operatorname{Tot} K_{*}\right) \quad \text { e } \quad E^{p+q} \Rightarrow \mathrm{H}^{p+q}\left(\operatorname{Tot} \mathrm{K}^{*}\right)
$$

As fórmulas dadas em $(*)$ induzem um produto cap nas sequências espectrais, isto é, quando $\omega=2,3, \ldots$, teremos:

$-\frown{ }_{-}: E_{r+s}^{\omega}(C) \otimes E_{\omega}^{p+q}(A) \longrightarrow E_{r-p, s-q}^{\omega}(C \otimes A)$

Por outro lado é conhecido o termo $\omega=2$ :

$$
\kappa_{*}: E_{r+s}^{2}(C) \stackrel{\cong}{\rightrightarrows} \mathrm{H}_{r}\left(Q ; \mathrm{H}_{s}(N ; C)\right) \quad \text { e } \quad \kappa^{*}: E_{2}^{p+q}(A) \stackrel{\cong}{\rightarrow} \mathrm{H}^{p}\left(Q ; \mathrm{H}^{q}(N ; A)\right)
$$

E através da homologia de $Q$ e $N$ também temos um produto cap abaixo induzido de maneira óbvia:

- $\frown$ - : $\mathrm{H}_{r}\left(Q ; \mathrm{H}_{s}(N ; C)\right) \otimes \mathrm{H}^{p}\left(Q ; \mathrm{H}^{q}(N ; A)\right) \rightarrow \mathrm{H}_{r-p}\left(Q ; \mathrm{H}_{s-q}(N ; C \otimes A)\right)$

Segue das definições acima uma demonstração trivial do:

Lema 2: O diagrama abaixo comuta a menos do sinal $(-1)^{s p}$ :

$$
\begin{aligned}
& E_{r+s}^{2}(C) \otimes E_{2}^{p+q}(A) \longrightarrow{ }^{2} \longrightarrow \\
& \kappa_{*} \otimes \kappa^{*} \downarrow \\
& H_{r}\left(Q ; H_{s}(\Lambda ; C)\right) \otimes H^{p}\left(Q ; H^{q}(N ; A)\right) \stackrel{\longrightarrow}{\longrightarrow} H_{r-p-q}\left(Q ; H_{s-q}(N ; C \otimes A)\right) \\
& \text { isto é, para todo } e \in E_{r+s}^{2}(C) \text { e } f \in E_{2}^{p+q}(A) \text { temos o seguinte: } \\
& \qquad \kappa_{*}(\epsilon \frown f)=(-1)^{s p} \kappa_{*}(e) \frown \kappa^{*}(f) .
\end{aligned}
$$

\section{II.2 - UM TEOREMA BÁSICO}

Neste parágrafo demonstraremos o seguinte Teorema: 
Teorema 1 : Seja $N \mapsto \mathrm{G} \rightarrow Q$ uma sequência exata curta de grupos. Se $N$ é de tipo (FP-n) e $Q$ é de tipo (FP-m) sobre um anel K, então $\mathrm{G}$ é de tipo (FP-(n+m)) sobre $\mathrm{K}$, resultando que $c d_{K} \mathrm{G}=c d_{K} N+c d_{K} Q$.

Antes de demonstrar este Teorema, precisamọ de alguns conceitos e resultados. Em primeiro lugar precisamos de um KG-isomorfismo

$$
\Psi_{*}: \mathrm{H}_{n}(\mathrm{G} ; B) \rightarrow \operatorname{Hom}_{\mathrm{G}}(C, B) \text { onde } B \text { e } C \text { são KG-módulos à direita. }
$$

Vamos então construí-lo:

Dado um KG-módulo $M$, seu dual $M^{*}$ é um KG-módulo à direita, definido como $M^{*}=\operatorname{Hom}_{G}(M, \mathrm{KG})$ com a KG-estrutura à direita induzida pela multiplicação à direita no anel de grupo KG. Seja $\mathrm{P} \rightarrow \mathrm{K}$ uma KG-resolução projetiva trivial do KG-módulo $\mathrm{K}$ e definimos:

(1) $\mathrm{h}_{k}(\mathrm{G}, B)=\mathrm{H}_{k}\left(\operatorname{Hom}_{\mathrm{G}}\left(\mathbf{P}^{*}, B\right)\right), \quad \forall \mathrm{KG}$-módulo à direita $B$. É fácil mostrar que esta definição independe da resolução $\mathrm{P}$.

Para qualquer KG-módulo à esquerda $M$, temos um homomorfismo natural $\Psi: B \otimes_{\mathrm{G}} M \rightarrow \operatorname{Hom}_{\mathrm{G}}\left(M^{*}, B\right)$ definido como:

$f \in M^{*}$ e $b \in B$.

$\Psi(b \otimes m)(f)=b \underbrace{f(m)}_{\in \mathrm{KG}} \in B$ (que é KG-módulo à direita), onde $m \in M$,

Como $\Psi$ é um homomorfismo natural ele induz um homomorfismo de complexos:

(2) $\Psi_{\#}: B \otimes_{\mathrm{G}} \mathbf{P} \rightarrow \operatorname{Hom}_{\mathrm{G}}\left(\mathbf{P}^{*}, B\right)$ e assim dos seus grupos de homologia:

(3) $\Psi_{*}: \mathrm{H}_{k}(\mathrm{G} ; B) \rightarrow \mathrm{h}_{k}(\mathrm{G} ; B), \quad \forall k \in \mathrm{K}$. 
3. Se M é um módulo projetivo FG, então (2) é um isomorfismo [21, V, proposição 4.2]. Segue que (3) é um isomorfismo se $\mathrm{K}$ admite uma $\mathrm{KG}$-resolução que seja FG em cada dimensão ( isto se aplica ao nosso caso, onde todos os grupos são dualidades).

4. Para grupos de dimensão cohomológica finita $\underline{\underline{n}}$, temos que:

$$
\begin{aligned}
& \mathrm{H}_{n}\left(H_{\mathrm{Gom}}\left(\mathrm{P}^{*}, B\right)\right) \cong \operatorname{Hom}_{\mathrm{G}}\left(\mathrm{H}^{n}\left(\mathrm{P}^{*}\right), B\right) \text {, ou seja, de (1) } \\
& \mathrm{h}_{n}(\mathrm{G} ; B) \cong \operatorname{Hom}_{G}\left(\mathrm{H}^{n}(\mathrm{G} ; \mathrm{KG}), B\right) .
\end{aligned}
$$

Assim. para grupos dualidades segue de (3), que:

$$
\Psi_{\text {. }} \mathrm{H}_{k}(\mathrm{G} ; B) \rightarrow \operatorname{Hom}_{G}\left(\mathrm{H}^{n}(\mathrm{G} ; \mathrm{KG}), B\right) \text { é um isomorfismo. }
$$

Notação: Quando queremos enfatizar o módulo coeficiente, como por exemplo no caso acima, escreveremos $\Psi_{*}^{B}$ para $\Psi_{*}$.

5. Seja $\mathrm{G}$ é um subgrupo normal num grupo $\overline{\mathrm{G}}, \mathrm{A}$ um $\mathrm{K} \overline{\mathrm{G}}-\mathrm{mó}$ dulo à esquerda e $\mathbf{P} \rightarrow \mathrm{K}$ uma $\mathrm{K} \overline{\mathrm{G}}$-resolução projetiva. A ação de um elemento $x \in \overline{\mathrm{G}}$ numa cocadeia $f \in \operatorname{Hom}_{G}(\mathbf{P}, A)$ e numa cadeia $b \otimes p \in B \otimes_{G} \mathbf{P}$ é dada por:

$\left(^{*}\right)\left\{\begin{array}{l}(x . f)(q)=x f\left(x^{-1} q\right), \quad \text { para todo } q \in \mathbf{P} \\ (b \supset p) \cdot x=b x \otimes x^{-1} p\end{array}\right.$

Uma $\overline{\mathrm{G}}$-ação em $\mathbf{P}^{*} \otimes A$ e em $\operatorname{Hom}_{G}\left(\mathbf{P}^{*}, B\right)$ é construida do seguinte modo:

Primeiro consideramos KG como um KG-módulo à esquerda por conjugação, usamos $\left({ }^{*}\right)$ para definir uma $\overline{\mathrm{G}}$-estrutura à esquerda em $\mathbf{P}^{*}$ e então convertemos isto numa $\overline{\mathrm{G}}$-estrutura à direita por:

$$
f x=x^{-1} f . \quad f \in \mathrm{P}^{*}, x \in \mathrm{G}
$$


(observemos que para elemento $x \in \mathrm{G}$ em particular, esta ação à direita em $\mathrm{P}^{*}$ coincide com a ação à direita induzida pela multiplicação à direita em KG).

Segundo, usamos a ação diagonal em $\otimes_{\mathrm{G}-\mathrm{e}} \operatorname{Hom}_{\mathrm{G}}(-,-)$ isto é, para qualquer $x \in \overline{\mathrm{G}}$ colocamos:

$$
\left(^{* *}\right)\left\{\begin{array}{l}
x .(. f \otimes a)=f x^{-1} \otimes x a, \quad f \in \mathbf{P}^{*}, x \in A \\
(F . x)(f)=F\left(f x^{-1}\right) x, \quad F \in \operatorname{Hom}_{G}\left(\mathbf{P}^{*}, B\right), f \in \mathbf{P}^{*}
\end{array}\right.
$$

Lema 1: Se $G$ é um subgrupo normal num grupo $\overline{\mathrm{G}}$ e $\mathrm{A}$ e $\mathrm{B}$ são $\mathrm{K} \overline{\mathrm{G}}$-módulos, então $\Psi_{*}$ é um $K \overline{\mathrm{G}}$-homomorfismo.

Demonstração: Seja $p \in \mathbf{P}, x \in \overline{\mathrm{G}}$ e $b \in B$. Então temos para todo $f \in \mathbf{P}^{*}$

$$
\begin{aligned}
& \Psi((b \otimes p) \cdot x)(f)=\Psi\left(b x \otimes x^{-1} p\right)(f)=b x f\left(x^{-1} p\right)=b x f\left(x^{-1} p\right) x^{-1} x= \\
& b(x f)(p) x=b\left(f x^{-1}\right)(p) x=\Psi((b \otimes p))\left(f x^{-1}\right) x=[\Psi(b \otimes p) \cdot x](f)
\end{aligned}
$$

Em $[11, I V, \S 6]$ obtem-se um homomorfismo $\alpha$ como segue:

Sejam $\mathrm{T}$ um funtor de duas variáveis, $A$ e $C$ complexos. Consideremos os seguintes diagramas:

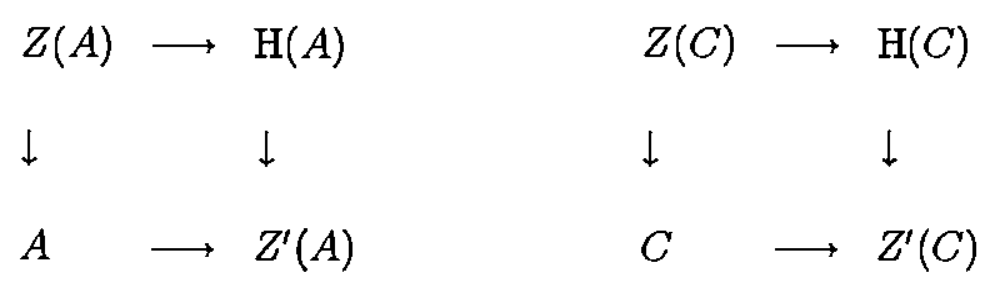

Aqui, $Z(A)=\operatorname{ker} d$ e $Z^{\prime}(A)=c o k e r d$, onde $d: A \rightarrow A$ é o homomorfismo diferenciação. Idem para $Z(C)$ e $Z^{\prime}(C)$. Estes dois diagramas induzem um diagrama comutativo: 


$$
\begin{array}{cc}
T\left(Z(A), Z^{\prime}(C)\right) \stackrel{\xi}{\longrightarrow} T(\mathrm{H}(A), \mathrm{H}(C)) \\
{ }^{\eta} & \downarrow^{\tau} \\
\mathrm{H}(T(A, C) & \underset{\varsigma}{\longrightarrow} T\left(Z^{\prime}(A), Z(C)\right)
\end{array}
$$

Lema 2: Se T é exato à direita, existe um único homomorfismo $\alpha: T(\mathrm{H}(A), \mathrm{H}(C)) \rightarrow$ $\mathrm{H}(T(A, C))$

Demostração do Teorema 1:

Ela se baseia essencialmente nos argumentos de [5, teorema 3.5], mas aqui olharemos mais cuidadosamente para uma classe fundamental de G.

Utilizaremos a seguinte notação:

$$
C_{N}=\mathrm{H}^{n}(N, \mathrm{KN}) ; \quad C_{Q}=\mathrm{H}^{m}(Q, \mathrm{~K} Q) ; \quad C=\mathrm{H}^{m+n}(\mathrm{G}, \mathrm{KG})
$$

Por argumentos de sequência espectral (dados em [5], na demonstração do teorema 3.5), obtemos um KG-isomorfismo $C \cong C_{Q} \otimes_{K} C_{N}$ (com ação diagonal do lado direito) e assim, naturalmente $c d_{K} G \leq n+m$.

Pelo Lema l e observação 4 acima, temos os KG-isomorfismos:

$$
\begin{aligned}
& \Psi_{*}^{C_{N}}: \mathrm{H}_{n}\left(N ; C_{N}\right) \cong \operatorname{Hom}_{N}\left(C_{N}, C_{N}\right) \\
& \qquad \Psi_{*}^{C_{Q}}: \mathrm{H}_{n}\left(Q ; C_{Q}\right) \cong \operatorname{Hom}_{Q}\left(C_{Q}, C_{Q}\right) . \\
& \text { Tomamos } e_{N}=\left(\Psi_{*}^{C_{N}}\right)^{-1}\left(I d_{C_{N}}\right) \text { e } e_{Q}=\left(\Psi_{*}^{C_{Q}}\right)^{-1}\left(I d_{C_{Q}}\right) \\
& \text { Consideremos a aplicação composta: } \\
& \qquad C_{Q} \stackrel{\varkappa}{\longrightarrow} C_{Q} \bigcirc_{K} \mathrm{H}_{n}\left(N ; C_{N}\right) \stackrel{\alpha}{\longrightarrow} \mathrm{H}_{n}\left(N ; C_{Q} \otimes_{K} C_{N}\right) \cong \mathrm{H}^{n}(N ; C) \text {, onde }
\end{aligned}
$$
$\varkappa$ é dado por $\varkappa(f)=f \otimes e_{N}, f \in C_{Q}$ e $\alpha$ sendo a aplicação funtorial descrita 
no lema 2, tomando-se o complexo $\mathrm{A}=H_{o} m_{Q}\left(\mathbf{P}_{Q} ; R Q\right)$ e o complexo $\mathrm{C}=$ $\mathbf{P}_{N} \otimes_{N} \operatorname{Hom}_{N}\left(\mathbf{P}_{N}, \mathrm{KN}\right)$ e como funtor $\mathrm{T}$ o produto tensorial sobre $\mathrm{K}$.

$\mathrm{A}$ ação de $\mathrm{G}$ em $e_{N}$ é trivial (usamos a ação diagonal em $\operatorname{Hom}_{N}\left(C_{N}, C_{N}\right)$ ), logo $\varkappa$ é um KG-isomorfismo. Temos também que $\alpha$ é um KG-homomorfismo, deste modo obtemos uma aplicação induzida:

$$
\alpha_{*} \varkappa_{*}: \mathrm{H}_{m}\left(Q ; C_{Q}\right) \longrightarrow \mathrm{H}_{m}\left(Q ; \mathrm{H}_{n}(N ; C)\right)
$$

Afirmamos que o produto cap com $e=\alpha_{*} \varkappa_{*}\left(e_{Q}\right)$ fornece um isomorfismo:

$$
e \frown \ldots: \mathrm{H}^{m}\left(Q ; \mathrm{H}^{n}(N ; A)\right) \stackrel{\cong}{\longrightarrow} \mathrm{H}_{0}\left(Q ; \mathrm{H}_{0}\left(N ; C \otimes_{\mathrm{K}} A\right)\right) \text { para todo KG- }
$$

módulo $A$ à esquerda.

De fato: Segue do diagrama comutativo abaixo:

* Notação:

Para facilitar a construção do diagrama utilizaremos a seguinte notação:

(i) $\mathrm{H}^{n}(N ; M)=C_{M}^{N}$ e (ii) $\mathrm{H}^{m}(Q ; M)=C_{M}^{Q}$.

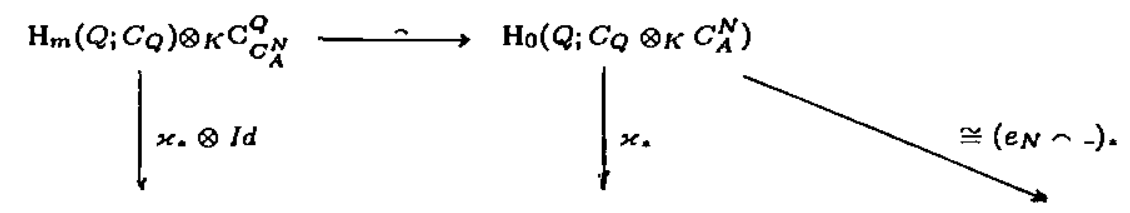

$\mathrm{H}_{m}\left(Q ; C_{Q} \otimes_{K} \mathrm{H}_{n}\left(N ; C_{N}\right)\right) \otimes_{K} C_{C_{A}^{N}}^{Q}=\mathrm{H}_{0}\left(Q ; C_{Q} \otimes_{K} \mathrm{H}_{n}\left(N ; C_{N}\right) \otimes_{K} C_{A}^{N}\right) \rightleftharpoons \mathrm{H}_{0}\left(Q ; C_{Q} \otimes_{K} \mathrm{H}_{0}\left(N ; C_{N} \otimes_{K} A\right)\right)$

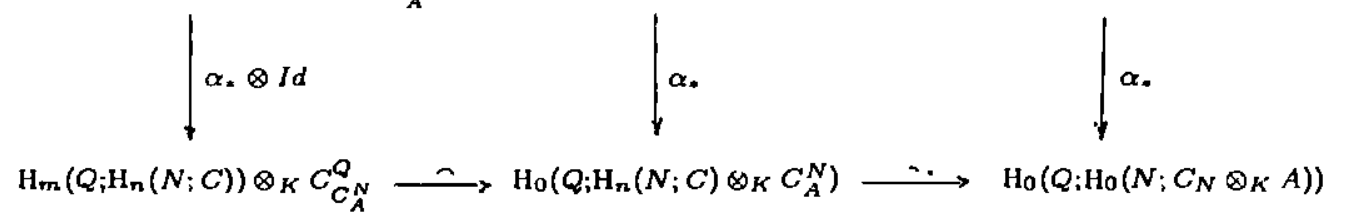

Novamente usando argumentos de sequência espectral, temos:

$$
\begin{aligned}
& \xi: \mathrm{H}_{m}\left(Q ; \mathrm{H}_{n}(N ; C)\right) \stackrel{\sim}{\rightarrow} \mathrm{H}_{m+n}(\mathrm{G} ; C) \\
& \eta: \mathrm{H}^{m}\left(Q ; \mathrm{H}^{n}(N ; A)\right) \stackrel{\sim}{\rightarrow} \mathrm{H}^{m+n}(\mathrm{G} ; A)
\end{aligned}
$$


O produto cap é compatível (a menos de sinal) com a sequência espectral de LHS (II.1), e assim também é compatível com $\xi$ e $\eta$. Segue que:

$$
(\xi(e) \frown-): \mathrm{H}^{m+n}(\mathrm{G} ; A) \rightarrow \mathrm{H}_{0}\left(\mathrm{G} ; C \otimes_{R} A\right) \text { é um isomorfismo.. }
$$

\section{II.3 - A DEMONSTRAÇÃO DO TEOREMA PRINCIPAL}

Vamos analisar $e=\alpha_{*} \varkappa_{*}\left(e_{Q}\right)$ a nível de cadeias.

Em [3, lema 1.3], mostra-se que se $\mathbf{X} \rightarrow \mathbb{Z}$ é uma $\mathbb{Z} G$-resolução livre de $\mathbb{Z}$ e $\mathbf{Y} \rightarrow \mathbb{Z}$ é uma $\mathbb{Z} Q$-resolução livre de $\mathbb{Z}$, então $\mathbf{P}=\operatorname{Tot}(\mathbf{Y} \otimes \mathbf{X})$ é uma $\mathbb{Z G}$ resolução livre de $\mathbb{Z}$, via o operador diagonal $\Delta: G \rightarrow Q \times G$, talque $\Delta(g)=$ $(\pi g, g)$

Podemos ver $\mathbf{X}$ como uma $\mathbb{Z} N$-resolução livre de $\mathbb{Z}$, via $i$. Então:

se $e_{N} \in \mathrm{H}_{n}\left(N ; C_{N}\right) \Rightarrow e_{N}=x \otimes_{N} c_{N}$, onde $x \in X_{n}$ e $c_{N} \in C_{N}$.

se $e_{Q} \in \mathrm{H}_{m}\left(Q ; C_{Q}\right) \Rightarrow e_{Q}=y \otimes_{Q} c_{Q}$, onde $y \in Y_{n}$ e $c_{Q} \in C_{Q}$.

Temos que:

$$
\varkappa_{*}\left(e_{Q}\right)=\varkappa_{*}\left(y \otimes_{Q} c_{Q}\right)=y \otimes_{Q} c_{Q} \otimes x \otimes_{N} c_{N} \text { (aqui está entrando o }
$$

fato de $N$ e $Q$ serem orientáveis, por que aqui estamos considerando fixadas as orientações).

$$
e=\alpha_{*} \varkappa_{*}\left(e_{Q}\right)=\alpha_{*} \varkappa_{*}\left(y \otimes_{Q} c_{Q}\right)=y \otimes_{Q}\left(x \otimes_{N} c_{Q} \otimes c_{N}\right)
$$

Com a urientação $e_{Q}$ fixa, definimos:

$$
j: C_{N} \rightarrow C_{N} \otimes C_{Q} \text {, colocando } j\left(c_{N}\right)=c_{N} \otimes c_{Q} \text {, onde } c_{Q} \text { é o elemento }
$$

de $C_{Q}$ que define a orientação $e_{Q}$. Assim temos uma aplicação induzida a nível de homologia: 
$j_{*}: \mathrm{H}_{n}\left(N ; C_{N}\right) \rightarrow \mathrm{H}_{n}(N ; C)$, dada por $j_{*}\left(x^{\prime} \otimes c_{N}\right)=x^{\prime} \otimes c_{N} \otimes c_{Q}$

A aplicação $i_{*}$ é a composta:

$$
\begin{gathered}
\mathrm{H}_{n}\left(N ; C_{N}\right) \stackrel{j_{*}}{\rightarrow} \mathrm{H}_{n}(N ; C) \stackrel{k_{*}}{\rightarrow} \mathrm{H}_{n}(G ; C) \\
x^{\prime} \otimes c_{N} \mapsto x^{\prime} \otimes c_{N} \otimes c_{Q} \mapsto i\left(x^{\prime}\right) \otimes c_{N} \otimes c_{Q} . \text { Portanto: } \\
\quad i_{*}\left(e_{N}\right)=i_{*}\left(x \otimes_{N} c_{N}\right)=x \otimes c_{N} \otimes c_{Q} .
\end{gathered}
$$

Vamos agora analisar $e_{G} \frown \pi^{*} \overline{e_{Q}}=\xi(e) \frown \pi^{*} \overline{e_{Q}}$

De acordo com II.1, o bicomplexo:

$$
\mathbf{K}_{s, r}(C)=\left(B_{r}(Q) \otimes B_{s}(G)\right) \otimes_{G} C \stackrel{\sim}{\longleftarrow} B_{r} Q \otimes_{Q}\left(B_{s}(G) \otimes_{N} C\right), \text { fornece }
$$

a sequência espectral . Aqui $B_{*}(\mathrm{G})$ e $B_{*}(Q)$ são resoluções $B$ ar de $\mathrm{G}$ e $Q$ respectivamente. $\mathrm{O}$ isomorfismo indicado acima nos fornece $\xi$.

$$
\therefore \xi(e)=\xi\left(y \otimes_{Q}\left(x \otimes c_{Q} \otimes c_{N}\right)\right)=(y \otimes x) \otimes_{\mathrm{G}}\left(c_{Q} \otimes c_{N}\right), \text { usando a }
$$

$\mathbb{Z} G$-resolução $\mathbf{P}$ descrita acima.

$\operatorname{Assim} \xi(e) \frown \pi^{*} \overline{e_{Q}}=\overline{e_{Q}}(y) \otimes x \otimes c_{Q} \otimes c_{N}$.

Pela observação $1 C_{Q} \cong C_{N} \cong C \cong \mathbb{Z}$. Então:

Pela observação $2 \overline{e_{Q}}(y)=1$, e assim concluímos o resultado. 


\section{CAPÍTULO III}

\section{O GRAU DE UMA APLICAÇÃO E $\odot$ NÚMERO DE UMA EXTENSÃO}

Em [19] Gottlieb definiu o grau e o cograu de uma aplicação $f$ entre espaços topológicos. Neste mesmo artigo, define-se o número fibrado ou o número da fibra de uma fibração $F \stackrel{i}{\mapsto} E \stackrel{p}{\rightarrow} B$. Aproveitamos a idéia aqui, para definirmos - grau de uma aplicação entre grupos e o número de uma extensão.

Utilizando estas definições, demonstramos vários resultados; para a demonstração de alguns deles utilizamos o teorema principal apresentado no capítulo II.

\section{III.1 - O GRAU DE UMA APLICAÇÃO}

Em I.6.1 definimos o grau de um homomorfismo entre grupos abelianos. Aqui definiremos o grau de um homomorfismo entre grupos quaisquer.

Definição:

Consideremos um homomorfismo $f: \mathrm{G}^{\prime} \rightarrow \mathrm{G}$, onde $\mathrm{G}^{\prime}$ e $\mathrm{G}$ são grupos quaisquer. O grau de $f$ denotado como $\operatorname{deg} f=\operatorname{deg} f_{*}$, onde $f_{*}: \mathrm{H}_{*}\left(\mathrm{G}^{\prime}\right) \rightarrow \mathrm{H}_{*}(\mathrm{G})$. 
O cograu de $f$ denotado como $\operatorname{cdg} f=\operatorname{cdg} f^{*}$, onde $f^{*}: \mathrm{H}^{*}(\mathrm{G}) \rightarrow \mathrm{H}^{*}\left(\mathrm{G}^{\prime}\right)$.

* Observações:

1. Se $f_{*}: \mathrm{H}_{*}\left(\mathrm{G}^{\prime} ; \mathrm{A}\right) \rightarrow \mathrm{H}_{*}(\mathrm{G} ; \mathrm{A})$, onde $\mathrm{A}$ é um módulo qualquer, escreveremos $\operatorname{deg}_{\mathrm{A}} f=\operatorname{deg} f_{*}$

2. $\operatorname{deg}_{\mathrm{A} \oplus \mathrm{B}} f=m m c\left\{\operatorname{deg}_{\mathrm{A}} f, \operatorname{deg}_{\mathrm{B}} f\right\}$.

De fato: $\left\{\begin{array}{l}\mathrm{H}_{*}\left(\mathrm{G}^{\prime} ; \mathrm{A} \oplus \mathrm{B}\right) \cong \mathrm{H}_{*}\left(\mathrm{G}^{\prime} ; \mathrm{A}\right) \oplus \mathrm{H}_{*}\left(\mathrm{G}^{\prime} ; \mathrm{B}\right) \\ \downarrow f_{*} \\ \mathrm{H}_{*}(\mathrm{G} ; \mathrm{A} \oplus \mathrm{B}) \cong \mathrm{H}_{*}(\mathrm{G} ; \mathrm{A}) \oplus \mathrm{H}_{*}(\mathrm{G} ; \mathrm{B})\end{array}\right.$

é um homomorfismo graduado. Assim o resultado segue da proposição 4 de I.6.2.

3. De maneira análoga definimos $\operatorname{cdg}_{\mathrm{A}} f$ como $\operatorname{cdg} f^{*}$, onde $f^{*}: \mathrm{H}^{*}(\mathrm{G} ; \mathrm{A}) \rightarrow$ $\mathrm{H}^{*}\left(\mathrm{G}^{\prime} ; \mathrm{A}\right)$ e mostra-se que $\operatorname{cdg}_{\mathrm{A} \oplus \mathrm{B}} f=m m c\left\{\operatorname{cdg}_{\mathrm{A}} f, \operatorname{cdg}_{\mathrm{B}} f\right\}$.

4. No caso particular em que $A=\mathbb{Z}_{r}$, escreveremos $\operatorname{deg}_{\mathbb{Z}_{r}}=\operatorname{deg}_{r}$ e $c \operatorname{cgg}_{\mathbb{Z}_{r}}=c \operatorname{cgg}_{r}$.

Teorema 1: Seja $f: G^{\prime} \rightarrow G$ uma aplicação de um grupo qualquer $\mathrm{G}^{\prime}$ num $\mathrm{PD}^{m_{-}}$ grupo orientável G. Então $(\operatorname{deg} f) e$ gera a imagem de $f_{*}$ em $\mathrm{H}_{m}(\mathrm{G})$, onde $e$ é a classe fundamental de G.

Demonstração: Temos que $\operatorname{Im} f_{*}$ é um subgrupo de $\mathrm{H}_{m}(\mathrm{G})$ que é cíclico infinito (Proposição 1 (iii) de I.4.1) gerado por $e$.

Suponhamos que $\beta e$ gera a imagem de $f_{*}$. Mostraremos que $\beta \backslash \operatorname{deg} f$ e $\operatorname{deg} f \backslash \beta$.

(a) $\beta \backslash \operatorname{deg} f$ Por definição de grau, existe um transfer $\tau, \operatorname{com} f_{* \circ} \tau=\operatorname{deg} f \quad \therefore$ $(\operatorname{deg} f) e$ está na imagem de $f_{*}$, pois $f_{*}(\tau(e))=(\operatorname{deg} f) e$. 
(b) $\operatorname{deg} f \backslash \beta$ Seja $\gamma \in \mathrm{H}_{m}\left(\mathrm{G}^{\prime}\right)$, tal que $f_{*}(\gamma)=\beta e$.

Definimos $\tau: \mathrm{H}_{*}(\mathrm{G}) \rightarrow \mathrm{H}_{*}\left(\mathrm{G}^{\prime}\right)$ colocando:

$$
\tau=(-\frown \gamma){ }_{\circ} f^{*}{ }_{\circ} D_{\mathrm{G}}^{-1} \text {, onde } D_{\mathrm{G}}^{-1}: \mathrm{H}_{i}(\mathrm{G}) \rightarrow \mathrm{H}^{m-i}(\mathrm{G}) \text { é a inversa da }
$$

aplicação dualidade de Poincaré.

Se $x \in \mathrm{H}_{i}(K) \Rightarrow \tau(x)=(-\frown \gamma) \circ f^{*} \circ D_{\mathrm{G}}^{-1}(x)=f^{*}(\underbrace{D_{\mathrm{G}}^{-1}(x)}) \frown \gamma \in \mathrm{H}_{i}\left(\mathrm{G}^{\prime}\right)$

$$
\underbrace{\in H^{m-i}(G)}_{\in H^{m-i}\left(G^{\prime}\right)}
$$

$\left(f_{* \circ} \tau\right)(x)=f_{*}\left(f^{*}\left(D_{\mathrm{G}}^{-1}(x)\right) \frown \gamma\right)=D_{\mathrm{G}}^{-1}(x) \frown f_{*}(\gamma)=D_{\mathrm{G}}^{-1}(x) \frown \beta e=$

$=\beta\left(D_{\mathrm{G}}^{-1}(x) \frown e\right)=\beta(x) \quad \therefore \quad \operatorname{deg} f \backslash \beta$.

* Observação:

5. Como G é um $\mathrm{PD}^{m}$-grupo orientável, o isomorfismo $\mathrm{H}^{k}(\mathrm{G} ; \mathrm{A}) \cong \mathrm{H}_{m-k}(\mathrm{G} ; \mathrm{A})$ é verdadeiro para todo $k$ e todo $\mathbb{Z G}$-módulo $\mathrm{A}$, assim a mesma demonstração do teorema acima, mostra que $\left(\operatorname{deg}_{r} f\right) e$ é gerador da imagem de $f_{*}: \mathrm{H}_{m}\left(\mathrm{G}^{\prime} ; \mathbb{Z}_{r}\right) \rightarrow$ $\mathrm{H}\left(\mathrm{G} ; \mathbb{Z}_{r}\right)$

Teorema 2: Suponhamos que $f: \mathrm{G}^{\prime} \rightarrow \mathrm{G}$ seja uma aplicação entre grupos tais que $\mathrm{G}^{\prime}$ é um $\mathrm{PD}^{m+n}$-grupo e $\mathrm{G}$ é um $\mathrm{PD}^{m}$-grupo. Então $\operatorname{deg}_{r} f=\operatorname{cdg}_{r} f$.

Demonstração: Definimos um homomorfismo $\bar{\tau}: \mathrm{H}^{*}\left(\mathrm{G}^{\prime} ; \mathbb{Z}_{r}\right) \rightarrow \mathrm{H}^{*}\left(\mathrm{G} ; \mathbb{Z}_{r}\right)$ colocando $\bar{\tau}(x) \frown e_{G}=f_{*}(x \frown \gamma)$ onde $\gamma \in \mathrm{H}_{m}\left(\mathrm{G}^{\prime} ; \mathbb{Z}_{r}\right)$ é tal que $f_{*}(\gamma)=\left(\operatorname{deg}_{r} f\right) e_{\mathrm{G}}$ (o Teorema 1 garante isso). Temos:

$\bar{\tau}\left(f^{*}(g)\right) \frown e_{\mathrm{G}}=f_{*}\left(f^{*}(g) \frown \gamma\right)=g \frown f_{*}(\gamma)=g \frown\left(\operatorname{deg}_{r} f\right) e_{\mathrm{G}}=$ $=\left(\operatorname{deg}_{r} f\right)\left(g \frown e_{\mathrm{G}}\right)$.

$\operatorname{Assim} \bar{\tau}_{\circ} f^{*}=\operatorname{deg}_{r} f . \operatorname{Logo} \operatorname{cdg}_{r} f \backslash \operatorname{deg}_{r} f$. 
Para mostrar que $\operatorname{deg}_{r} f \backslash \operatorname{cdg}_{r} f$, vamos utilizar o TCU no caso em que $\mathrm{G}^{\prime}$ é um $\mathrm{PD}^{m+n}$-grupo.

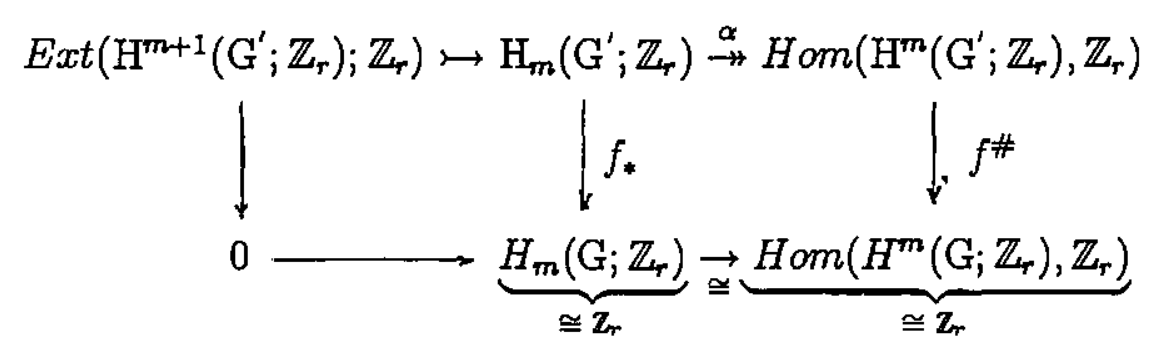

Seja $\bar{\tau}: \mathrm{H}^{m}\left(\mathrm{G}^{\prime} ; \mathbb{Z}_{r}\right) \rightarrow \mathrm{H}^{m}\left(\mathrm{G}^{\prime} ; \mathbb{Z}_{r}\right)$ um cotransfer associado ao $\operatorname{cdg}_{n} f$. Assim $\bar{\tau} \in \operatorname{Hom}\left(\mathrm{H}^{m}\left(\mathrm{G}^{\prime} ; \mathbb{Z}_{r}\right) ; \mathbb{Z}_{r}\right)$.

Consideremos $\tau \in \mathrm{H}_{m}\left(\mathrm{G}^{\prime} ; \mathbb{Z}_{\tau}\right)$ um elemento tal que $\alpha(\tau)=\bar{\tau}$. Então:

$f_{*}(\tau)=f^{\#}(\bar{\tau})=\widetilde{\tau}_{\circ} f^{*}=\operatorname{cdg}_{n} f \therefore \operatorname{cdg}_{n} f \in \operatorname{Im} f_{*}$ que é gerada por $\left(\operatorname{deg}_{n} f\right) e_{G}$ pelo Teorema 1. Assim $\operatorname{deg}_{n} f \backslash \operatorname{cdg}_{n} f$.

\section{III.2 - O NÚMERO DE UMA EXTENSÃO}

Seja $N \stackrel{i}{\rightarrow} \mathrm{G} \stackrel{p}{\rightarrow} Q$ uma extensão de grupos e suponhamos que $\mathrm{H}^{j}(N)=0$, para todo $j>n$ e que $\mathrm{H}^{n}(N) \cong \mathbb{Z}$. Assim a imagem de $i^{*}: \mathrm{H}^{n}(\mathrm{G}) \rightarrow \mathrm{H}^{n}(N)$ é um subgrupo de $\mathbb{Z}$ e assim tem um gerador não-negativo $\Phi$.

\section{Definição:}

$\Phi=\Phi(p)$ é o número da extensāo do grupo $N$ pelo grupo $Q$.

* Observação:

1. Trocando-se o coeficiente $\mathbb{Z}$ por $\mathbb{Z}_{r}$, obteremos $\Phi_{r}(p) \in \mathbb{Z}$ o número da extensão do grupo $N$ pelo grupo $Q$ para coeficientes em $\mathbb{Z}_{r}$. O menor inteiro positivo tal que $\left(\Phi_{r}\right) g$ gera $\operatorname{Im} i^{*} \subset \mathrm{H}^{n}\left(N ; \mathbb{Z}_{r}\right)$, onde $g$ é a classe fundamental em $\mathrm{H}^{n}\left(N ; \mathbb{Z}_{r}\right)$ no sentido dado na observação 2 no início deste capítulo. 
Definição:

Dizemos que a extensão de grupo $N \stackrel{i}{\mapsto} \mathrm{G} \stackrel{p}{\rightarrow} Q$ é orientável se $Q$ atua trivialmente em $\mathrm{H}^{n}(N)$

Teorema 1: $\Phi \neq 0 \Rightarrow$ a extensão é orientável.

Demonstração: Suponhamos que a ação não é trivial, então para qualquer $\alpha \in$ $H^{n}(N), \alpha \neq 0, \exists g \in Q$, tal que $g \alpha=-\alpha$.

Assim $\left(\mathrm{H}^{n}(N)\right)^{Q}=\{0\}$, ou seja $\mathrm{H}^{0}\left(Q, \mathrm{H}^{n}(N)\right)=\{0\}$.

Mas $i^{*}$ é dada pela composta:

$$
\mathrm{H}^{n}(\mathrm{G}) \rightarrow E_{\infty}^{0, n} \rightarrow E_{2}^{0, n} \cong \mathrm{H}^{0}\left(Q, H^{n}(N)\right)=\{0\}, \text { ou seja, a imagem de } i^{*}
$$

localiza-se na parte invariante de $\mathrm{H}^{n}(N)$ sob $Q$, que é nula. Assim $\Phi=0$.

Na sequència denotaremos $\mathbb{Z}$ por $\mathbb{Z}_{0}$.

Suponhamos que $N \stackrel{i}{\stackrel{1}{\leftrightarrow}} \mathrm{G} \stackrel{p}{\rightarrow} Q$ seja uma extensão orientada. Então a sequência espectral de Hochschild-Serre, nos fornece as aplicações:

(1) $p^{\#}: \mathrm{H}^{i}\left(\mathrm{G} \cdot \mathbb{Z}_{r}\right) \rightarrow E_{\infty}^{i-n, n} \mapsto E_{2}^{i-n, n} \cong \mathrm{H}^{i-n}\left(Q, H^{n}\left(N ; \mathbb{Z}_{r}\right)\right) \cong H^{i-n}\left(Q ; \mathbb{Z}_{r}\right)$

(2) $p_{\#}: \mathrm{H}_{i}\left(Q, \mathbb{Z}_{r}\right) \cong \mathrm{H}_{i}\left(Q, \mathrm{H}_{n}\left(N, \mathbb{Z}_{r}\right)\right) \cong E_{i, n}^{2} \rightarrow E_{i, n}^{\infty} \mapsto \mathrm{H}_{i+n}\left(\mathrm{G}, \mathbb{Z}_{r}\right)$

* Observação:

2. A compatibilidade dos produtos cup e cap com as sequências espectrais de Hochschild-Serre, além da naturalidade dos produtos, fornecem os diagramas comutativos (a menos de sinal) abaixo: 


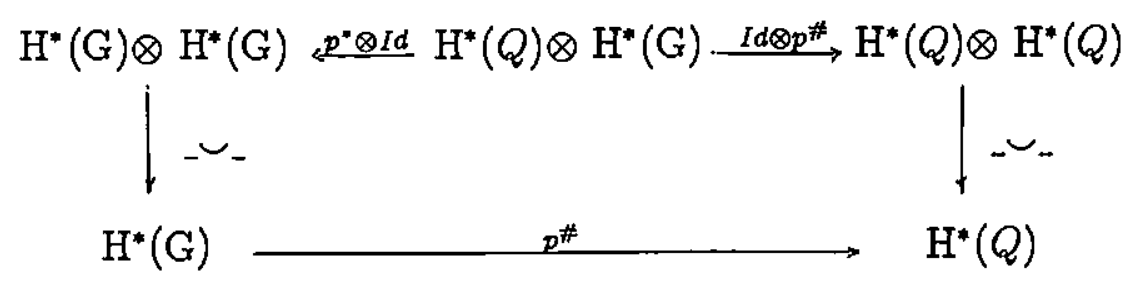

diagrama 1 .

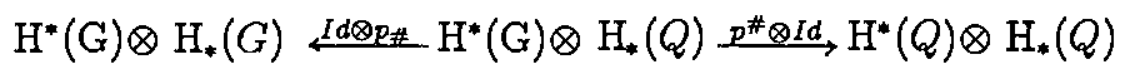

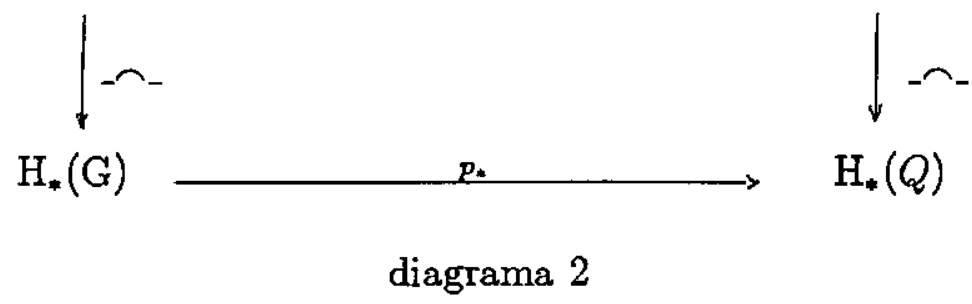

ou seja, $p^{\#}$ e $p_{\#}$ satisfazem a menos de sinal as equações:
(a) $p^{\#}\left(p^{*} x \smile y\right)=x \smile p^{\#}(y)$
(b) $p_{*}\left(y \frown p_{\#} \xi\right)=p^{\#} y \frown \xi$.

Se $N$ e $Q$ são respectivamente $\mathrm{PD}^{n}$-grupo e $\mathrm{PD}^{m}$-grupo orientáveis, temos pelo teorema 2 de I.4.1 que $\mathrm{G}$ é um $\mathrm{PD}^{n+m}$-grupo e se a extensão é orientável então $\mathrm{G}$ é orientável.

De agora em diante estaremos trabalhando sob estas hipóteses.

Vamos definir dois homomorfismos $p_{!}: \mathrm{H}_{i}(Q) \rightarrow \mathrm{H}_{n+i}(\mathrm{G})$ e $p^{!}: \mathrm{H}^{i}(\mathrm{G}) \rightarrow$ $\mathrm{H}^{\imath-n}(Q)$, de tal modo que os diagramas 3 e 4 abaixo comutem:

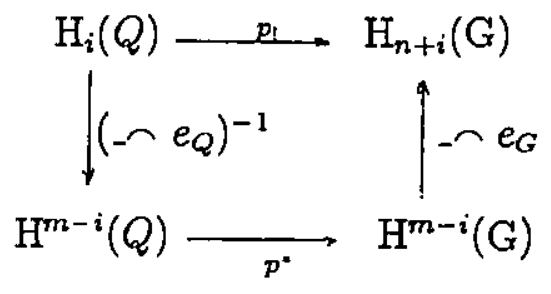

diagrama 3

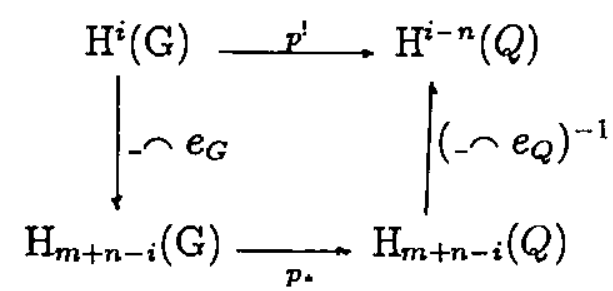

diagrama 4 
onde $e_{G}$ e $e_{Q}$ são classes fundamentais de $\mathrm{G}$ e $Q$ respectivamente.

Lema 1: As seguintes igualdades se verificam:

1. $p_{!}\left(y \frown e_{Q}\right)=p^{*}(y) \frown e_{\mathrm{G}}$.

2. $p^{\prime}(y) \frown e_{Q}=p_{*}\left(y \frown e_{\mathrm{G}}\right)$.

Demonstração:

1. $p_{\mathrm{l}}\left(y \frown e_{Q}\right)=\left[\left(-\frown e_{\mathrm{G}}\right) \circ p^{*} \circ\left(-\frown e_{Q}\right)^{-1}\right]\left(y \frown e_{Q}\right)=\left(-\frown e_{\mathrm{G}}\right) \circ p^{*}(y)=$ $=p^{*}(y) \frown e_{\mathrm{G}}$.

2. $p^{\prime}(y) \frown e_{Q}=\left[\left(-\frown e_{Q}\right)^{-1} \circ p_{*} \circ\left(-\frown e_{\mathrm{G}}\right)\right](y) \frown e_{Q}=$ $\left[\left(-e_{Q}\right)^{-1} \circ p_{*}\left(y \frown e_{\mathrm{G}}\right)\right] \frown e_{Q}=\left(p_{*}\left(y \frown e_{\mathrm{G}}\right) \frown e_{Q}\right)^{-1} \frown e_{Q}=p_{*}\left(y \frown e_{\mathrm{G}}\right) \cdot \mathrm{m}$

Lema 2: Pelo Teorema 1 de II.2 temos que $p_{\# \#} e_{Q}=e_{\mathrm{G}}$ e pela observação 2 (b) $p^{\#} e_{\mathrm{G}}=e_{Q}$, .disso segue que:

1. $p^{\#}=p^{!}$e $2 . p_{\#}=p_{\text {! }}$

Demonstração:

1. $p^{\#}(y) \frown e_{Q}=p_{*}\left(y \frown p_{\#}\left(e_{Q}\right)\right)=p_{*}\left(y \frown e_{G}\right)=p^{l}(y) \frown e_{Q}$.

2. análoga a 1 .

Teorema 2: $\operatorname{deg}_{n} p \backslash \Phi_{n}(p)$ e $\operatorname{cdg}_{n} p \backslash \Phi_{n}(p)$.

Demonstração: Seja $\lambda \in \mathrm{H}^{n}(\mathrm{G})$ tal que $i^{*}(\lambda)=\Phi_{n} \overline{e_{N}} \in \mathrm{H}^{n}(N)$, onde $\overline{e_{N}}$ é a classe fundamental em $\mathrm{H}^{n}(N)$, isto é, $\overline{e_{N}} \frown e_{N}=1$.

Definimos os homomorfismos:

(1) $\tau: \mathrm{H}_{i}(Q) \rightarrow \mathrm{H}_{i}(\mathrm{G})$, colocando $\tau(\alpha)=\lambda \frown p_{\#}(\alpha)$

(2) $\bar{\tau}: \mathrm{H}^{i}(\mathrm{G}) \rightarrow \mathrm{H}^{i}(Q)$, colocando $\bar{\tau}(x)=p^{\#}(x \smile \lambda)$ 
Mas $p^{\#}: \mathrm{H}^{n}\left(\mathrm{G}, \mathbb{Z}_{r}\right) \rightarrow \mathrm{H}^{0}\left(Q, H^{n}\left(N ; \mathbb{Z}_{r}\right)\right)$ é tal que:

(3) $p^{\#}(\lambda)=\Phi_{n} \cdot 1$.

Assim:

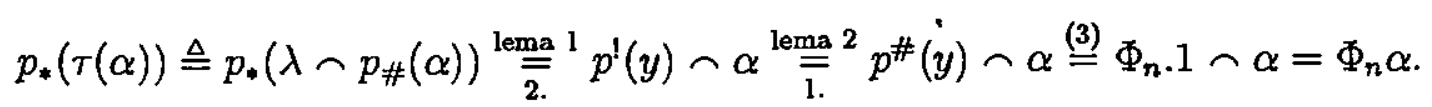

$\bar{\tau}\left(p^{*}(x)\right) \triangleq p^{\#}\left(p^{*}(x) \smile \lambda\right)=x \smile p^{\#}(\lambda) \stackrel{(3)}{=} x \smile \Phi_{n} .1=\Phi_{n} x$.

Portanto $\tau$ e $\bar{\tau}$ são transfers associados a $p_{*}$ e $p^{*}$ respectivamente. Logo temos:

$$
\operatorname{deg}_{n} p \backslash \Phi_{n}(p) \quad \text { e } \quad \operatorname{cdg}_{n} p \backslash \Phi_{n}(p)
$$

Lema 3: Seja $N \stackrel{i}{\mapsto} \mathrm{G} \stackrel{p}{\rightarrow} Q$ uma extensão de grupo orientável com $N$ um $\mathrm{PD}^{n}$. grupo e $Q$ um $\mathrm{PD}^{m}$-grupo, então $p^{*}\left(\overrightarrow{e_{Q}}\right)=i^{\prime}(1)$, onde $\overline{e_{Q}}$ é a classe fundamental de $\mathrm{H}^{m}(Q)$.

Demonstração: Consideremos o diagrama comutativo abaixo:

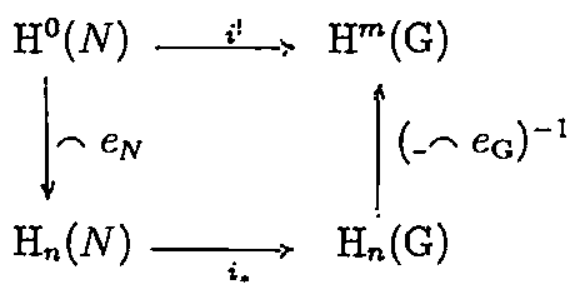

diagrama 5

$$
\begin{aligned}
& i^{!}(1)=\left[\left(\frown e_{\mathrm{G}}\right)^{-1} \circ i_{* \circ} \frown e_{N}\right](1)=\left(\frown e_{\mathrm{G}}\right)^{-1}\left(i_{*}\left(e_{N}\right)\right) \\
& =\left(\frown e_{\mathrm{G}}\right)^{-1}\left(p^{*}\left(\overline{e_{Q}}\right) \frown e_{\mathrm{G}}\right)=p^{*}\left(\overline{e_{Q}}\right)
\end{aligned}
$$

Lema 4. : Seja $N \stackrel{i}{\rightarrow} \mathrm{G} \stackrel{p}{\rightarrow} Q$ uma extensão de grupo orientável com $N$ um $\mathrm{PD}^{n_{-}}$ grupo e $Q$ um $\mathrm{PD}^{m}$-grupo. Pelo Teorema 1 de II.4, existe $\gamma \in \mathrm{H}_{*}\left(\mathrm{G}, \mathbb{Z}_{r}\right)$ tal que 
$p_{*}(\gamma)=\operatorname{deg}_{n}(p) e_{Q}$ e a dualidade de Poincaré garante que existe $\bar{\gamma} \in \mathrm{H}^{*}\left(\mathrm{G}, \mathbb{Z}_{r}\right)$ tal que $\gamma=\bar{\gamma} \frown e_{G}$. Então: $i_{1}\left(\bar{\gamma} \frown e_{\mathrm{G}}\right)=i^{*}(\bar{\gamma}) \frown e_{N}$.

Demonstração: Consideremos o diagrama comutativo abaixo:

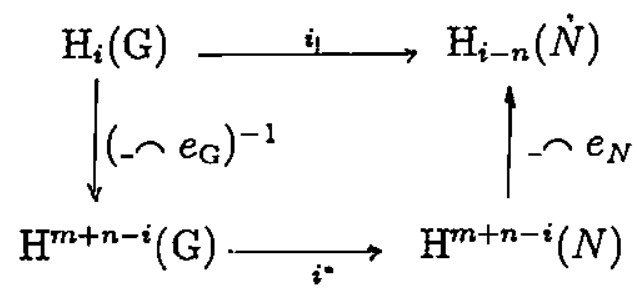

diagrama 6

$i_{1}\left(\bar{\gamma} \frown e_{G}\right)=\left[\left(\frown e_{N}\right) \circ i^{*} \circ\left(-\frown e_{N}\right)^{-1}\right]\left(\bar{\gamma} \frown e_{G}\right)=\left(\frown e_{N}\right)\left(i^{*}(\bar{\gamma})\right)=i^{*}(\bar{\gamma}) \frown e_{N}$

Teorema 3 : Seja $N \stackrel{i}{\rightarrow} \mathrm{G} \stackrel{p}{\rightarrow} Q$ uma extensão de grupo orientável com $N$ um $\mathrm{PD}^{n}$-grupo e $Q$ um $\mathrm{PD}^{m}$-grupo, então $\operatorname{deg}_{n}(p)=\Phi_{n}(p)=\operatorname{cdg}_{n}(p)$.

Demonstração: Pelo Teorema 2, temos que $\operatorname{deg}_{n} p \backslash \Phi_{n}(p)$. Mostremos que $\Phi_{n}(p) \backslash \operatorname{deg}_{n} p$.

Pelo Teorema 1 de II.4, existe $\gamma \in \mathrm{H}_{*}\left(\mathrm{G}, \mathbb{Z}_{r}\right)$ talque $p_{*}(\gamma)=\operatorname{deg}_{n}(p) e_{Q}$.

Seja $\overline{e_{Q}}$ a classe fundamental de $\mathrm{H}^{m}(Q)$, ou seja $\overline{e_{Q}} \frown e_{Q}=1$.

A dualidade de Poincaré garante que existe $\bar{\gamma} \in \mathrm{H}^{*}\left(\mathrm{G}, \mathbb{Z}_{r}\right)$ tal que $\gamma=\bar{\gamma} \frown$ $e_{G}$. Então:

$\operatorname{deg}_{n} p \triangleq\left\langle\overline{e_{Q}} \cdot p_{*}(\gamma)\right\rangle=\left\langle p^{*}\left(\overline{e_{Q}}\right), \gamma\right\rangle \stackrel{\text { lema } 3}{=}\left\langle i^{1}(1), \gamma\right\rangle=\left\langle 1, i_{!}(\gamma)\right\rangle \therefore i_{!}(\gamma)=\left(\operatorname{deg}_{n} p\right) .1$

Agora:

$\operatorname{deg}_{n} p=i_{!}(\cdot)=i_{!}\left(\bar{\gamma} \frown e_{G}\right) \stackrel{\text { lema }}{=}{ }^{4} i^{*}(\bar{\gamma}) \frown e_{N}=k \overline{e_{N}} \frown e_{N}=k$, onde $k$ é um inteiro tal que $i^{*}(\bar{\gamma})=k \overline{e_{N}}$. 


\section{BIBLIOGRAFIA}

[1]- Andrade, M.G.C. - "Tese de Doutorado" - IMECC - UNICAMP, Campinas(1992)

[2]- Atiyah, M.F. - "The sgnature of fibre-bundles" - Univ. Tokyo Press, Tokyo (1969)73-84.

[3]- Beyl, F.F. - "The spectral sequence of a group extension" - Bull. Sc. Math, $2^{a}$ série, 105 (1981)417-434.

[4]- Bieri, R. - "Gruppen mit Poincaré Dualität" - Comment. Math. Helv., 47 (1972)373-396.

[5]- Bieri, R and Eckmann, B - "Groups with homological duality generalizing Poincaré duality" - Invent. Math., 20(1973) 103-124.

[6]- Bieri, R. - "On groups of finite cohomological dimension and Duality Groups over a ring" - J. Pure Appl. Algebra, 6 (1975)83-109.

[7]- Bieri, R. - "Homological Dimension of Discrete Groups" - Queen Mary College Math. Notes, London, 1976.

[8]- Bieri, R. and Eckmann, B. - "Cobordism for Poincaré Duality Groups" - Bull. AMS, 82, nº 1 (1976)137-139.

[9]- Bieri, R. and Eckmann, B. - "Relative Homology and Poincaré Duality for Groups Pairs" - J. Pure App. Algebra, 13 (1978)277-319.

[10]- Brown, K.S. - "Cohomology of Groups" - Springer-Verlag - GTM, 87 (1982).

[11]- Cartan, H. and Eilemberg, S.- "Homological Algebra" - Princiton University Press (1957). 
Manifold" -Trans. AMS, 74(1953)110-134.

[13]- Dicks, W. and Dunwood, M. - "Groups Acting on Graphs" - Cambridge University Press (1988).

[14]- Eckmann, B and Müller, H. "Poincaré Duality Groups of Dimension Two" - Comm. Math. Helv. 55(1980) 510-520.

[15]- Eckmann, B and Lionell, P.A - "Poincaré Duality Groups of Dimension Two II" - Comm. Math. Helv. 58(1983) 111-114.

[16]- Evens, L. - "The Cohomology of Groups" - Clarendon Press (1991).

[17]- Fanti, E.L.C. - "Tese de Doutorado"- ICMSC-USP- São Carlos (1992).

[18]- Gottlieb, D.L - "Partial Transfers" - LNM,657 - Springer Verlag (1977) $255-266$.

[19]- Gottlieb, D.L - "The Trace an action and the degree of a map" - Trans. AMS 293(1986) 381-410.

[20]- Hochschild, G. and Serre, J.P. - "Cohomology of Group Extensions" - Trans. Amer. Math. Soc. 74(1953)257-309.

[21]- Kodaira, K. - "A certain type of Irregular Algebraic Surfaces" - J. Anal. Math IXX (1967)207-215.

[22]- Mac Lane, S. - "Homology" - Math. Wissensch, 114 - Springer, Berlin (1967).

[23]- Penteado, D. - "Índice de uma variedade fibrada" - Dissertação de mestrado - ICMSC/USP - São Carlos (1979).

[24]- Robinson, D.J.S - "A Course in the Theory of Groups" - GTM 80 - SpringerBerlin (1982). 
[25]- Spanier, E.H. "Albebraic Topology" - M,Graw-Hill, New York (1966).

[26]- Strebel, R. - "A Remark on Subgroups of Infinite Index in Poincaré Duality

Groups" - Comm. Math. Helv. (1977)317-324.

[27]- Vick, J.W. - "Homology Theory" - Academic Press (1973).

[28]- Whitehead, G. W. - "Homotopy Theory" - The M.I.T. Press, Massachusetts (1966). 